\title{
Disruption of Gpr45 causes reduced hypothalamic POMC expression and obesity
}

\author{
Jing Cui, ${ }^{1}$ Yi Ding, ${ }^{1}$ Shu Chen, ${ }^{1}$ Xiaoqiang Zhu, ${ }^{1}$ Yichen Wu, ${ }^{1}$ Mingliang Zhang, ${ }^{2}$ Yaxin Zhao, ${ }^{1}$ Tong-Ruei R. Li, ${ }^{1}$ Ling V. Sun, ${ }^{1}$ \\ Shimin Zhao, ${ }^{1}$ Yuan Zhuang, ${ }^{1,3}$ Weiping Jia, ${ }^{2}$ Lei Xue, ${ }^{1}$ Min Han, ${ }^{1,4}$ Tian Xu, ${ }^{1,5}$ and Xiaohui Wu' \\ 1State Key Laboratory of Cenetic Engineering and National Center for International Research of Development and Disease, Institute of Developmental Biology and Molecular Medicine, Collaborative \\ Innovation Center of Genetics and Development, School of Life Sciences, Fudan University, Shanghai, China. ${ }^{2}$ Department of Endocrinology and Metabolism, Shanghai Jiao Tong University Affiliated Sixth \\ People's Hospital, Shanghai Clinical Medical Center of Diabetes, Shanghai Key Clinical Center of Metabolic Diseases, Shanghai Institute of Diabetes, Shanghai Key Laboratory of Diabetes, Shanghai, China. \\ ${ }^{3}$ Department of Immunology, Duke University Medical Center, Durham, North Carolina, USA. ${ }^{4}$ Howard Hughes Medical Institute, Department of Molecular, Cellular, and Developmental Biology, \\ University of Colorado, Boulder, Colorado, USA. ${ }^{5}$ Howard Hughes Medical Institute, Department of Cenetics, Yale University School of Medicine, New Haven, Connecticut, USA.
}

\begin{abstract}
A rise in the occurrence of obesity has driven exploration of its underlying genetic basis and potential targets for intervention. CWAS studies have identified obesity susceptibility pathways involving several neuropeptides that control energy homeostasis, suggesting that variations in the genes that regulate food intake and energy expenditure may contribute to obesity. In this study, we identified 5 additional obesity loci, including a neuronal orphan GPCR called Gpr45, in a forward genetic screen of mutant mice generated by piggyBac insertional mutagenesis. Disruption of Gpr45 led to increased adiposity at the time of weaning and increases in body mass, fat content, glucose intolerance, and hepatic steatosis with advancing age. Mice with disruptions in Gpr45 also displayed a reduction in expression of the metabolic regulator POMC and less energy expenditure prior to the onset of obesity. Mechanistically, we determined that CPR45 regulates POMC expression via the JAK/STAT pathway in a cell-autonomous manner. Consistent with this finding, intraventricular administration of melanotan-2, an analog of the POMC derivative $\alpha$-MSH, suppressed adult obesity in Gpr45 mutants. These results reveal that CPR45 is a regulator of POMC signaling and energy expenditure, which suggests that it may be a potential intervention target to combat obesity.
\end{abstract}

\section{Introduction}

As a rapidly growing health threat in modern society, obesity can result from a shifted balance toward more food intake and less energy expenditure $(1,2)$. It is estimated that genetic factors contribute up to $70 \%$ of obesity, but only a small fraction of obese patients have been identified as sufferers of defined obese mutations (3). For example, the melanocortin 4 receptor (MC4R) has been shown to cause morbid obesity in mice when mutated (4), and is recognized as one of the most commonly affected loci in obese patients. However, MC4R mutations only account for $1 \%-4 \%$ of obesity cases $(5-7)$. Therefore, the mechanisms by which energy balance is shifted upon encountering different stimuli remain to be explored; more efforts are needed in the search for genes that regulate energy balance and obesity.

The central nervous system (CNS) plays pivotal roles in energy balance regulation. Many obesity susceptibility genes identified by genome-wide association studies (GWAS) in humans are abundantly expressed in the CNS, especially in the hypothalamus

Note regarding evaluation of this manuscript: Manuscripts authored by scientists associated with Duke University, The University of North Carolina at Chapel Hill, Duke-NUS, and the Sanford-Burnham Medical Research Institute are handled not by members of the editorial board but rather by the science editors, who consult with selected external editors and reviewers.

Conflict of interest: The authors have declared that no conflict of interest exists. Submitted: November 23, 2015; Accepted: June 9, 2016.

Reference information: / Clin Invest. 2016;126(9):3192-3206. doi:10.1172/JCI85676.
$(8,9)$. A number of neuropeptides expressed in the CNS, such as the agouti-related peptide (AGRP), neuropeptide Y (NPY), proopiomelanocortin (POMC), cocaine- and amphetamine-related transcript (CART), corticotropin-releasing hormone (CRH), thyrotropin-releasing hormone (TRH), brain-derived neurotrophic factor (BDNF), melanin-concentrating hormone (MCH), and orexin, have been shown to be critical regulators of energy homeostasis (10-12). Among them, the POMC-mediated pathway is the most extensively studied. In the hypothalamus, POMC is produced by POMC neurons in the arcuate nucleus (Arc). It is then sequentially cleaved to generate adrenocorticotropin (ACTH), $\alpha-, \beta-, \gamma$-melanocyte-stimulating hormones ( $\alpha-, \beta-, \gamma-\mathrm{MSHs})$, and endogenous opioid $\beta$-endorphin (13). Secreted $\alpha$-MSH transduces signals through melanocortin receptors, MC3R and MC4R, in various nuclei of the brain to inhibit food intake and to stimulate energy expenditure (14). POMC signaling can be regulated by various central and peripheral stimuli. For example, adipocyte-derived leptin stimulates the expression of POMC $(15,16)$. Depletion of POMC neurons in the Arc, as well as mutations in leptin (Lep), the leptin receptor (Lepr), Pomc, $M c 3 r$, or $M c 4 r$, leads to extra fat accumulation in mice $(4,17-22)$. Mutations of these genes have also been detected in obese patients (23-29). Thus, the POMC-mediated pathway may serve as a valuable candidate target for obesity intervention.

Genetically modified mice have made significant contributions to biomedical studies $(30,31)$. Phenotype-driven screening 
of mutant collections allows for unbiased identification of genetic contributors to a specific cellular or developmental process; such an approach has great potential to expand our knowledge of the underlying mechanisms. We have generated the PBmice collection of more than 5,000 mutant mice, each carrying a single-copy piggyBac $(P B)$ insertion in a known or predicted gene $(32,33)$. In this report, we describe a pilot genetic screen for obese mutations in PBmice that uncovered an orphan $\mathrm{G}$ protein-coupled receptor (GPCR), GPR45, as a novel POMC regulator.

\section{Results}

A pilot genetic screen identifies obese mutations. We designed a 2-round screening strategy for obese mutants. The primary screen focused on body weight increase, which was followed by body composition analysis in the secondary screen. Mutants with confirmed obese or overweight phenotypes were also tested for blood glucose alterations. We performed a pilot study with 408 randomly selected $P B$ lines (Figure 1A). Although body weights of sampled lines exhibited a continuous distribution, 14 lines (3.4\%) with significantly heavier body weight at the age of 12 weeks were successfully identified from the primary screen (Figure 1, A and B, and Supplemental Table 1; supplemental material available online with this article; doi:10.1172/JCI85676DS1). At the same time, we noticed a leptin receptor mutant $\left(\right.$ Lepr $\left.^{P B}\right)$ during identification of reported metabolism regulators in PBmice. These 15 lines were chosen for the second round of analysis (Figure 1B). Compared with littermate controls, 7 lines showed a higher fat/ lean ratio and thus were recognized as obese mutants (Figure 1, C and D). One line ( $D g k h)$ showed significantly higher body weight, but showed no increase in the fat/lean ratio. Among the genes affected in obese mutants, Lepr and the follicle-stimulating hormone receptor ( $F s h r$ ) have been previously reported to cause obesity when mutated in mice $(21,34)$. Obesity-related phenotypes have not been previously described to be associated with the other 5 loci, indicating the potential value of PBmice in searching for new obesity-related genes.

Disrupting Gpr45 expression in PB mutants results in obesity, hepatic steatosis, and impaired glucose homeostasis. Among the 5 newly discovered loci, an insertion upstream of the gene encoding an orphan GPCR, Gpr45, caused the most dramatic obese phenotypes in mutants (35) (Figure 2A). The genomic sequence of Gpr45 is composed of 2 exons. In the identified mutant, an intronic $P B$ insertion ( $\left.G p r 45^{P B 1}\right)$ was mapped at 34,038 bp upstream of the only coding exon (Figure 2B). This insertion effectively disrupted the expression of Gpr45. Quantitative analysis of 5-day-old (P5) mice revealed a nearly complete loss (97.3\%) of Gpr45 mRNA in the homozygous mutants and a 55.2\% reduction in heterozygous animals (Figure 2C). Adult Gpr45 mutants also displayed hepatic steatosis, a condition that often develops in obese individuals. Livers of 6-month-old female and male Gpr $45^{P B 1 / P B 1}$ mice were $14.4 \%$ and $32.2 \%$ heavier than those of wild-type littermates, respectively (Figure 2D). Oil red O staining detected prominent accumulation of fat lipid droplets in the liver of 6-month-old mutants (Figure 2E).

Compared with wild-type littermates, $G p r 45^{P B 1 / P B 1}$ mice started to gain extra weight beginning at 6 weeks of age (Figure 2F). At the age of 11 weeks, homozygous female and male mice were $33.6 \%$ and $16.9 \%$ heavier, respectively. NMR analysis revealed that 5-week-old Gpr45 $5^{P B 1 / P B 1}$ mice already exhibited a marked increase in body fat content, though their average weight was still normal. Specifically, the fat/lean ratios in female and male mice of that age were 1.3 and 1.2 times higher than those of wild-type littermates, respectively (Figure 2G). Meanwhile the lean mass of mutants decreased $6.4 \%$ and $9.3 \%$ in females and males, respectively (Figure $2 \mathrm{H}$ ). At the age of 12 weeks, the fat/lean ratios in female and male $G p r 45^{P B 1 / P B 1}$ mice increased to 4.3 and 4.9 times those of the wild-type littermates, respectively (Figure $2 \mathrm{G}$ ). In contrast, female and male mutants lost $5.8 \%$ and $9.8 \%$ lean mass, respectively (Figure 2H). Consistent with these observations, 6-month-old $G p r 45^{P B 1 / P B 1}$ mice had significantly larger fat tissues than control mice; the weight of perigonadal and retroperitoneal fat pads in homozygous female mutants increased by 3.1 and 4.0 times over those of wild-type littermates, respectively. Their interscapular brown fat pads were also 2.2 times heavier. In male $G p r 45^{P B 1 / P B 1}$ mice of the same age, perigonadal, retroperitoneal, and interscapular fat pads were 2.1, 2.3, and 1.5 times heavier, respectively (Figure 2I). Heterozygous mutants had similar body weight and lean mass as their wild-type littermates when they were young (Figure 2, $\mathrm{F}$ and $\mathrm{H}$ ), but displayed a reduction in their lean mass as adults (Figure $2 \mathrm{H}$ ). In addition, male and young female $\mathrm{Gpr} 45^{\mathrm{PBI} / \mathrm{+}}$ heterozygous mice had slightly higher fat/lean ratios (Figure $2 \mathrm{G}$ ).

Most reported obesity cases are accompanied with high plasma leptin, while high plasma insulin is often observed in obese and type 2 diabetic patients (36-39). We thus examined the leptin and insulin levels in the blood of obese Gpr45 mutants. Indeed, we observed that fasting plasma leptin levels in female and male mutants were 9.9 and 23.8 times higher than those of the wild-type littermates, respectively (Figure 3, A and D). At the same time, fasting plasma insulin levels in female and male mutants were 1.5 and 2 times higher, respectively (Figure 3, B and E). These data suggest that disruption of Gpr45 leads to hyperleptinemia and hyperinsulinemia in mice.

Hyperinsulinemia is usually recognized as a sign of insulin resistance that could lead to impaired glucose homeostasis in type 2 diabetes. We thus analyzed the insulin sensitivity of 15 -week-old Gpr $45^{P B 1 / P B 1}$ mice. Insulin tolerance tests (ITTs) revealed that both female and male mutants not only exhibited significantly higher blood glucose, but also showed less efficient glucose clearance upon receiving insulin (Figure 3, C and F). Consistent with this observation, hyperinsulinemic-euglycemic clamp analysis also revealed a $66 \%$ reduction in the glucose infusion rate in 15 -weekold male mutants (Figure 3I). These results indicate that Gpr45 mutants suffer from insulin resistance.

Hyperglycemia observed in free-fed mutants also suggested impaired glucose homeostasis caused by the Gpr45 mutation. To test this idea, we performed glucose tolerance tests (GTTs) in 12 -week-old mutants. Fasted for 16 hours, both female and male Gpr $45^{P B 1 / P B 1}$ mice showed significantly higher blood glucose levels than wild-type littermates (female, $7.33 \pm 0.22 \mathrm{mM}$ vs. $6.12 \pm$ $0.21 \mathrm{mM}, P<0.001$; male, $7.15 \pm 0.29 \mathrm{mM}$ vs. $6.29 \pm 0.12 \mathrm{mM}, P<$ 0.05). After receiving an intraperitoneal injection of glucose, the blood glucose level of mutant mice increased more quickly and cleared more slowly than that of the wild-type mice (Figure 3, G and $\mathrm{H}$ ). The lean mass normalized area under the glucose tolerance curve (nAUC) in mutants is higher than that of wild-type lit- 
A

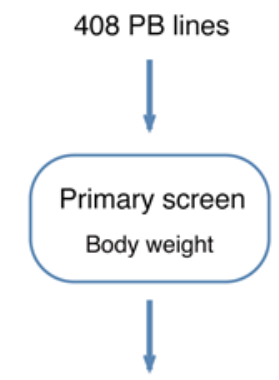

14 overweight

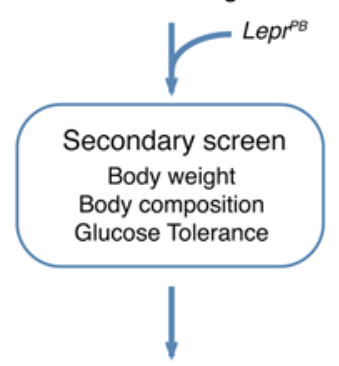

7 obese

1 overweight

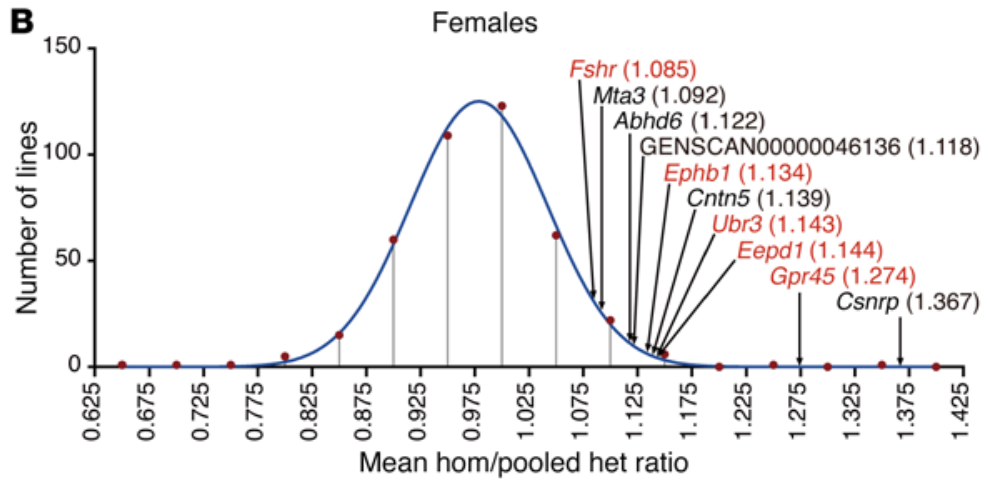

Males

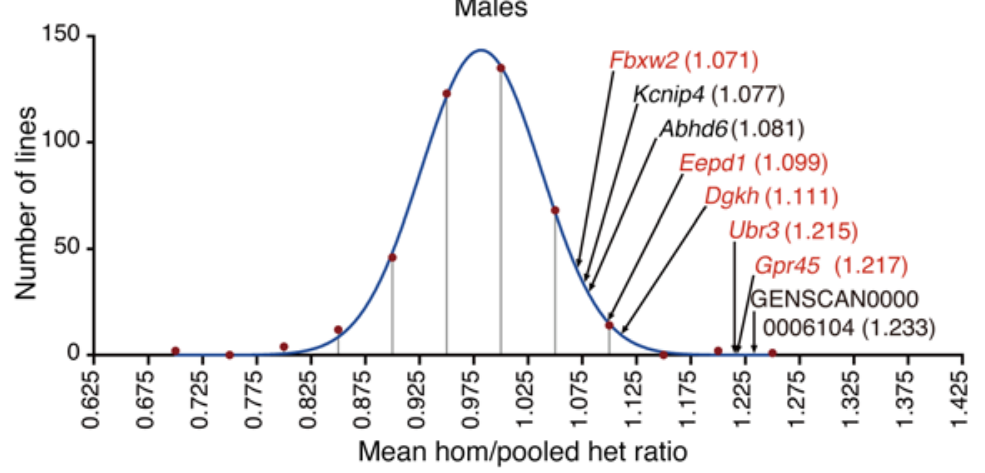

C

\begin{tabular}{|c|c|c|c|c|c|}
\hline Gene & Sex & BW & F/L & FPG & AUC \\
\hline \multirow{2}{*}{ Lepr } & $\mathrm{F}$ & $225 \%$ & $1169 \%$ & $302 \%$ & \\
\cline { 2 - 6 } & $\mathrm{M}$ & $163 \%$ & $763 \%$ & $313 \%$ & \\
\hline \multirow{2}{*}{ Gpr45 } & $\mathrm{F}$ & $131 \%$ & $428 \%$ & $120 \%$ & $162 \%$ \\
\cline { 2 - 6 } & $\mathrm{M}$ & $111 \%$ & $491 \%$ & $114 \%$ & $160 \%$ \\
\hline \multirow{2}{*}{ Ubr3 } & $\mathrm{F}$ & $110 \%$ & $164 \%$ & $113 \%$ & $113 \%$ \\
\cline { 2 - 6 } & $\mathrm{M}$ & $104 \%$ & $178 \%$ & $103 \%$ & $114 \%$ \\
\hline \multirow{3}{*}{ Fbxw2 } & $\mathrm{F}$ & $110 \%$ & $118 \%$ & $110 \%$ & $124 \%$ \\
\cline { 2 - 6 } & $\mathrm{M}$ & $105 \%$ & $173 \%$ & $90 \%$ & $130 \%$ \\
\hline \multirow{2}{*}{ Fshr } & $\mathrm{F}$ & $104 \%$ & $156 \%$ & $140 \%$ & $138 \%$ \\
\cline { 2 - 6 } & $\mathrm{M}$ & $99 \%$ & $89 \%$ & $75 \%$ & $94 \%$ \\
\hline \multirow{2}{*}{ Ephb1 } & $\mathrm{F}$ & $114 \%$ & $153 \%$ & $94 \%$ & $105 \%$ \\
\cline { 2 - 6 } & $\mathrm{M}$ & $103 \%$ & $93 \%$ & $94 \%$ & $103 \%$ \\
\hline \multirow{2}{*}{ Eepd1 } & $\mathrm{F}$ & $103 \%$ & $136 \%$ & $88 \%$ & $88 \%$ \\
\cline { 2 - 6 } & $\mathrm{M}$ & $103 \%$ & $84 \%$ & $100 \%$ & $100 \%$ \\
\hline \multirow{2}{*}{ Dgkh } & $\mathrm{F}$ & $100 \%$ & $98 \%$ & $100 \%$ & $111 \%$ \\
\cline { 2 - 6 } & $\mathrm{M}$ & $111 \%$ & $116 \%$ & $107 \%$ & $109 \%$ \\
\hline \multirow{2}{*}{} & & & &
\end{tabular}

High Low NS
D

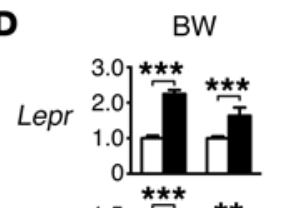

$\mathrm{F} / \mathrm{L}$

FPG
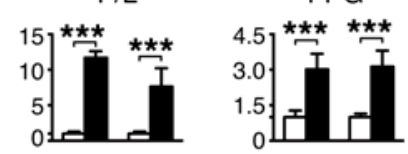

Gpr45
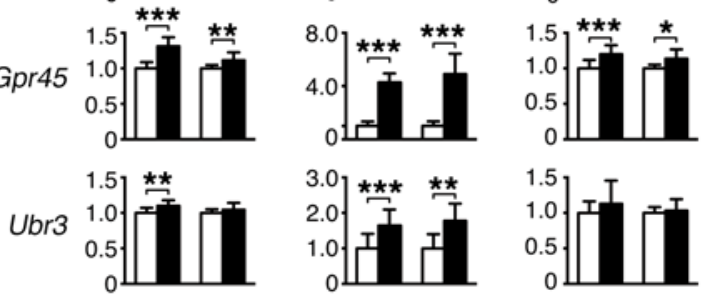

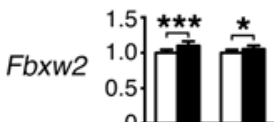
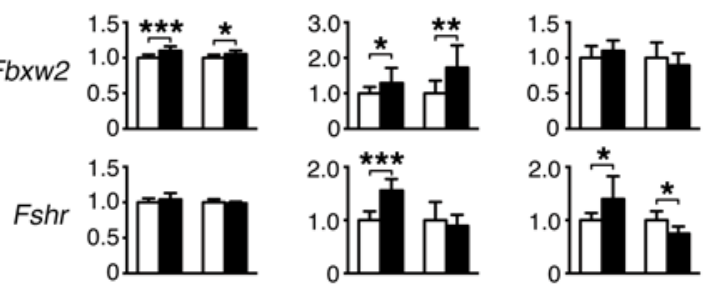

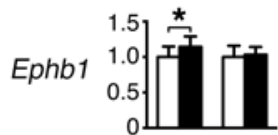
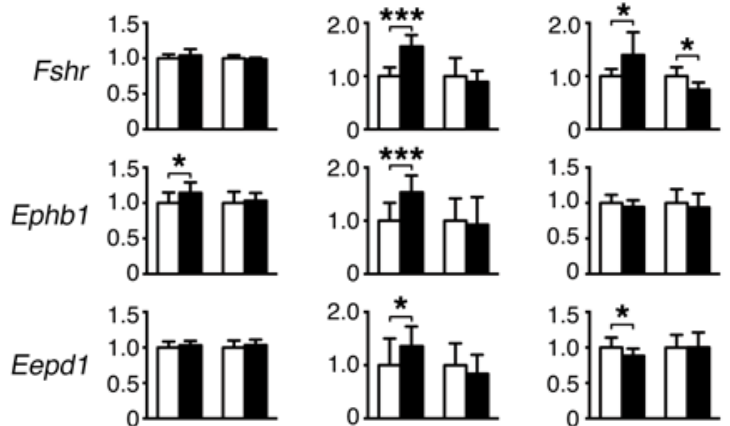

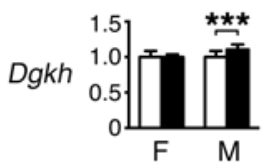

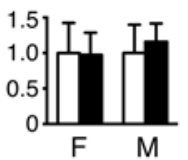

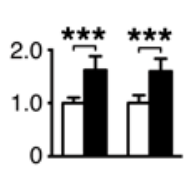

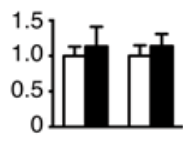

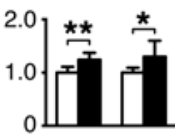
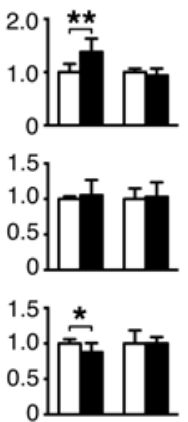

AUC

마++

- $\mathrm{PB} / \mathrm{PB}$

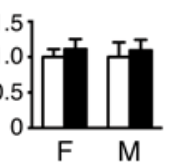


Figure 1. A pilot genetic screen for obese mutations. (A) The screen pipeline. (B) Body weight distribution of screened mutants. Numbers of lines with homozygotes/heterozygotes (hom/het) body weight ratio in a given range are illustrated as red dots. The histogram data were normalized by Gaussian distribution and plotted as an overlay (blue line). Hom/het body weight ratios of overweight mutants used in the secondary screen are also shown on the overlay in red (obese or overweight) or black (nonobese or nonoverweight). (C) Body weight (BW), fat/lean ratio ( $F / L)$, fasted plasma glucose (FPG) and the area under the glucose tolerance test curve (AUC) for obese and overweight lines are compared with those of the wild-type littermates and shown as a heat map. Red, significantly high; blue, significantly low; no color, no significant difference. (D) Histogram presentations of fold changes in BW, F/L, FPG, and AUC of both 12-week-old mutants and their wild-type littermates, the values of which were arbitrarily defined as 1 . Asterisks indicate statistically significant differences calculated with raw numbers. Empty bars, $+/+(n \geq 3)$; filled bars, $\mathrm{PB} / \mathrm{PB}(n \geq 3)$. All data are shown as the mean $\pm \mathrm{SEM} .{ }^{*} P<0.05$, ${ }^{* *} P<0.01$, and ${ }^{* * *} P<0.001$ by Student's $t$ test.

termates (females, $1,488 \pm 62.8 \mathrm{mM} \cdot \mathrm{min}$ vs. $1,281 \pm 41.7 \mathrm{mM} \cdot \mathrm{min}$, $P<0.05$; males, $1,785 \pm 90.3 \mathrm{mM} \cdot \mathrm{min}$ vs. $1,378 \pm 70.3 \mathrm{mM} \cdot \mathrm{min}$, $P<0.01$ ) (Figure 3, G and H). These data indicate that the ability to regulate blood glucose level is compromised in Gpr 45 mutants.

By searching the PBmice database, we identified another mutant carrying a $P B$ insertion $\left(G p r 45^{P B 2}\right) 20,428$ bp upstream of $G p r 45^{P B 1}$ in the same intron (Figure 2B). This mutation behaves similarly to $G p r 45^{P B 1}$. Compared to wild-type littermates, Gpr45 expression in Gpr $45^{P B 2 / P B 2}$ mice was reduced by $89.4 \%$ (Supplemental Figure 1A). Adult Gpr45 $5^{P B 2 / P B 2}$ mice had much higher body weight and fat/lean ratios than wide-type littermates, but no alterations in lean mass (Supplemental Figure 1, B-D). Impaired blood glucose homeostasis was also observed in 12-week-old Gpr45 $5^{\text {PB2/PB2 }}$ mice by GTTs (Supplemental Figure 1, E and F).

Similar metabolic defects observed in both $G p r 45^{P B 1}$ and $G p r 45^{P B 2}$ mutants indicate a causative role of Gpr 45 disruption in these mice. To confirm this conclusion, mice with a revertant allele of $G p r 45^{P B 1}$ were produced by precise removal of the inserted $P B$ transposon (Supplemental Figure 2A). As expected, mice homozygous for the revertant allele (Gpr45 $\left.5^{\mathrm{rev} / \mathrm{rev}}\right)$ had body weight, fat/lean ratio, and lean mass similar to those of wild-type animals (Figure 2, F-H and Supplemental Figure 2, B-D). In addition, both female and male revertants showed normal glucose tolerance capacity in GTTs (Supplemental Figure 2, E and F). These data are consistent with the conclusion that the observed metabolic defects in $P B$ mutants are caused by disruption of the Gpr45 locus. Therefore, we used $G p r 45^{P B 1}$ for further studies.

Obesity is the primary physiological defect in Gpr45 mutants. Defects in lipid and glucose metabolism have profound influences on each other. For example, obesity increases the risk of insulin resistance and type 2 diabetes (39), while insulin resistance of skeletal muscle could lead to fat accumulation in mice $(40,41)$. Furthermore, hepatic steatosis contributes to both insulin resistance and obesity-related diseases $(42,43)$. To determine the primary physiological role of GPR 45 with regard to the multiple physiological defects described above, we compared the onset of multiple metabolic abnormalities in Gpr $45^{P B 1 / P B 1}$ mice.

We first followed the initiation of obesity. As mentioned above, $G \operatorname{pr} 45^{P B 1 / P B 1}$ mice had more fat accumulation at the age of 5 weeks, though their body weight was still comparable with that of the wild- type littermates (Figure 2, F and G). To identify the age of onset of obesity, we monitored the body composition of $G p r 45^{P B 1 / P B 1}$ mice at P10 to P28. Although mutants had a comparable fat/lean ratio at the beginning, they stored fat more efficiently than their wildtype littermates. As a result, female and male mutants became obese from P2O and P18, respectively (Figure 4A). Consistent with these changes, the fasted plasma leptin level of $G p r 45^{P B 1 / P B 1}$ mice was comparable with that of the wild-type littermates at P14, but increased by 1.3 and 1.6 times in females and males 2 weeks later, respectively (Figure 4, B and C). These results indicate that obesity develops in $G p r 45^{P B 1 / P B 1}$ mice around weaning.

In contrast to obesity, hepatic steatosis and impaired glucose homeostasis were not detected in young Gpr45 mutants. We did not observe fat accumulation in the livers of 4-week-old $G p r 45^{P B 1 / P B 1}$ mice (Figure 4D). At the same age, homozygous mutants and wildtype mice had similar fasted insulin levels (Figure 4E). They also showed comparable levels of fasted blood glucose at time points as late as 9 weeks of age (Figure $4 \mathrm{~F}$ ). Considering the facts that obesity could lead to both hepatic steatosis and impaired glucose homeostasis, and that obesity appears ahead of other defects in Gpr45 mutants, we conclude that obesity is the primary physiological defect upon the disruption of Gpr45.

Disruption of Gpr45 decreases energy expenditure. Obesity is the consequence of energy imbalance due to increased energy intake and/or decreased energy expenditure (EE). To better understand the mechanism underlying the obesity in Gpr45 mutants, we analyzed both processes before or at the early stage of obesity.

We first examined the alterations of energy intake in $G p r 45^{P B 1 / P B 1}$ mice by measuring food intake and fecal calories. We found that the Gpr 45 mutation did not affect food intake at the early stage of obesity. Specifically, analysis of covariance (ANCOVA) of animals between P21 and P33 revealed comparable amounts of food intake between Gpr $45^{P B 1 / P B 1}$ mice and their wild-type littermates (Figure 5A). In addition, no difference in fecal calorie measurements was found between 3-week-old Gpr45 $5^{P B 1 / P B 1}$ mice and their wild-type littermates (Supplemental Figure 3A). These results suggest that disrupting Gpr45 does not affect energy intake at the early stage of obesity.

We then examined the EE of Gpr $45^{P B 1 / P B 1}$ mice. We first measured 24-hour real-time EE of animals between P21 and P33 at room temperature $\left(22^{\circ} \mathrm{C}\right)$ by indirect calorimetry (Supplemental Figure 3, C and D). ANCOVA revealed that $G p r 45^{P B 1 / P B 1}$ mice had significantly lower 24-hour total EE estimated in the common lean mass than their wild-type littermates (female, $8.87 \pm 0.12$ kcal vs. $9.94 \pm 0.13 \mathrm{kcal}, P<0.001$; male $8.78 \pm 0.14$ kcal vs. $9.74 \pm$ $0.18 \mathrm{kcal}, P<0.001$ ) (Figure 5B). Respiratory quotient, an indicator of substrate utilized to produce energy, was not significantly altered in Gpr45 $5^{P B 1 / P B 1}$ mice at P21 to P33 (Supplemental Figure 3B). These results suggest that disrupting Gpr45 reduces EE without affecting the choice of energy sources at the time around the initiation of obesity.

EE consists of energy spent by physical activity, basal metabolism, and adaptive thermogenesis (44). To examine which of these processes contributes to reduced $\mathrm{EE}$, we measured the related physiological parameters in the Gpr 45 mutants.

Physical activity consumes approximately $40 \%$ of the energy of laboratory mice (45). To evaluate whether altered physical activity contributes to decreased EE in Gpr45 mutants, we measured their 
A

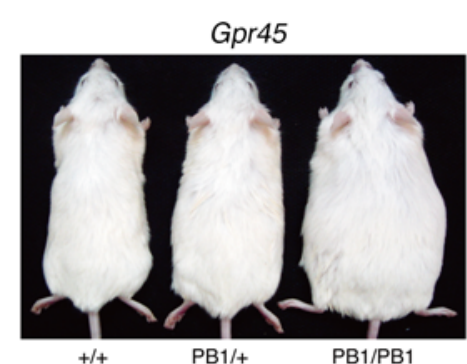

B

Gpr45
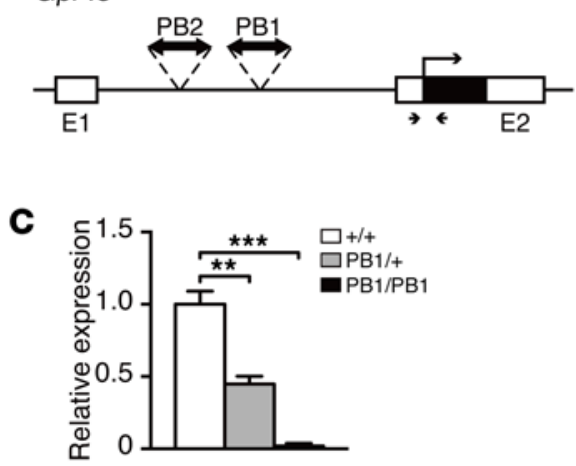

D

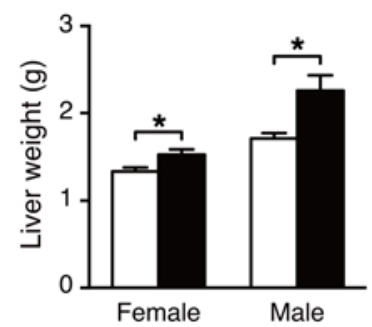

E

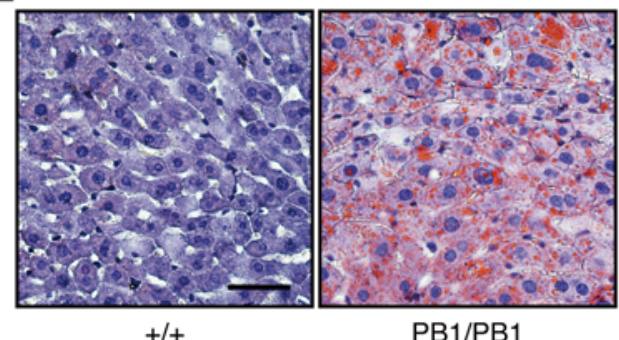

$\mathbf{F}$

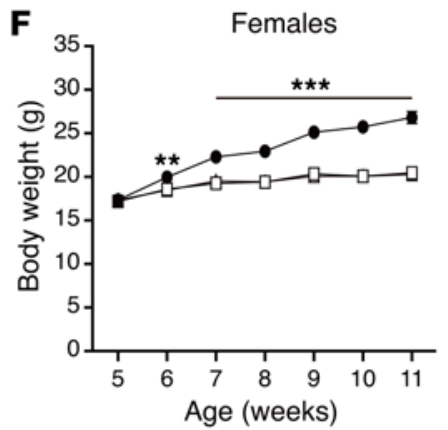

G

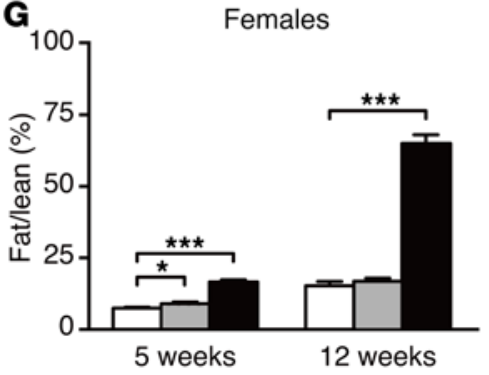

H
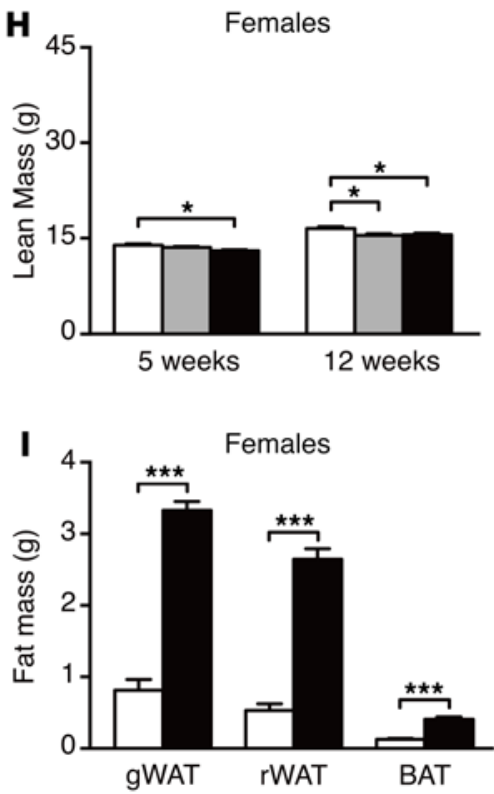

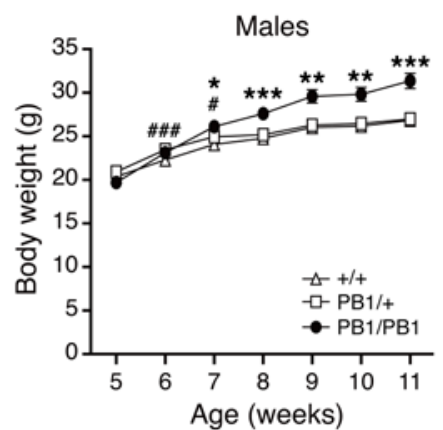

Males

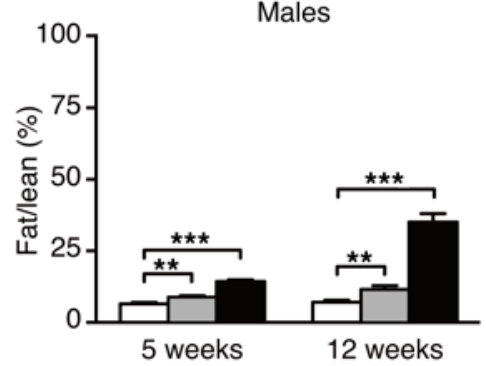

Males
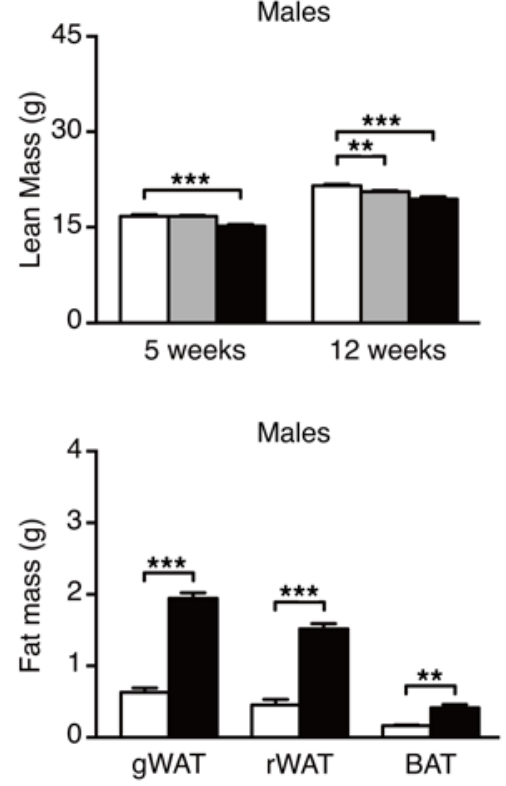

Figure 2. Disruption of Gpr45 causes obesity. (A) Photographs of three 1-year-old littermates of indicated Gpr45 genotypes. (B) Cartoon illustration of the position of 2 independent $P B$ insertions in Gpr45. Both insertions are mapped in the only intron of Gpr45, upstream of its coding exon (E2). The filled black box indicates the coding sequence. Arrowheads indicate the position of primers for real-time RT-PCR analysis. (C) Real-time RT-PCR analysis showing decreased Gpr45 expression in brains of P5 mutant mice. Empty bars, $+/+(n=5)$; dashed bars, PB1/+ $(n=4)$; filled bars, PB1/PB1 ( $n=4)$. Wild-type data served as the statistical controls. The bar patterns of genotypes also applies to $\mathbf{D}, \mathbf{G}, \mathbf{H}$ and $\mathbf{I}$. (D) Average liver mass of 6 -month-old females $(+/+, n=8$; PB1/PB1, $n=9)$ and males $(+/+, n=4$; PB1/PB1, $n=4)$. (E) Representative Oil red 0 and hematoxylin staining showing hepatic steatosis in a 6 -month-old male mouse. Scale bar: $0.05 \mathrm{~mm}$. (F) Body weight curves of female $(+/+, n=15 ; \mathrm{PB} 1 /+, n=22$; PB1/PB1, $n=16)$ and male $(+/+, n=10 ; \mathrm{PB} 1 /+, n=16 ; \mathrm{PB} 1 /$ PB1, $n=14)$ littermates. Wild-type data served as the statistical controls. ( $\mathbf{G}$ and $\mathbf{H})$ Average fat/lean ratio (G) and lean mass $(\mathbf{H})$ of $G p r 45$ littermates ( $n \geq 10$ for each group at each time point). (I) Average fat pad mass of 6-month-old female (+/+, $n=8$; PB1/PB1, $n=9)$ and male (+/+, $n=4$; PB1/PB1, $n=4)$ mice. gWAT, perigonadal fat; rWAT, retroperitoneal fat; BAT, interscapular brown fat. All data are shown as the mean $\pm S E M$. ${ }^{*} P<0.05$, ${ }^{* *} P<0.01$ and ${ }^{* *} P<0.001$ by Student's $t$ test; ${ }^{\#} P<0.05$ and ${ }^{\# \#} P<0.001$, PB1 $/+$ versus $+/+$ in $\mathbf{F}$.

travel and sleep performance at the age of 3 weeks. HomeCageScan analysis revealed that female and male $G p r 45^{P B 1 / P B 1}$ mice traveled $47.6 \%$ and $29.7 \%$ less, but slept $96 \%$ longer than their wild-type littermates (Figure 5C). Similar tendencies were also observed in
Gpr $45^{P B 1 / P B 1}$ mice at the age of 7 to 9 weeks (Supplemental Figure 3, E and F). Furthermore, SHIRPA analysis did not detect major neurological or musculoskeletal abnormalities in either 4- or 8-week-old Gpr45 $5^{P B 1 / P B I}$ mice (Supplemental Table 2). Collectively, these data 
A Females (12 weeks)

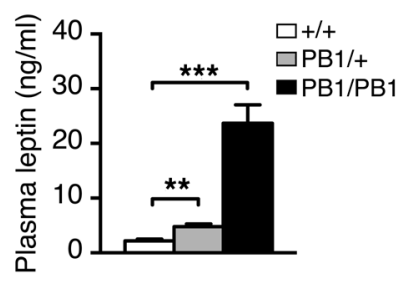

D Males (12 weeks)

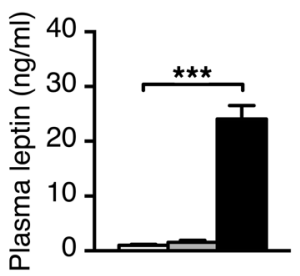

G

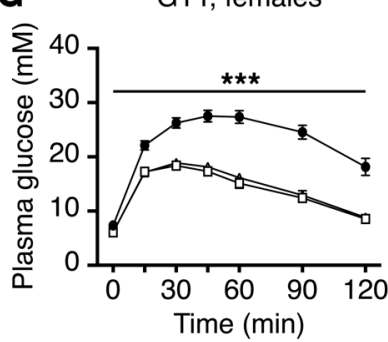

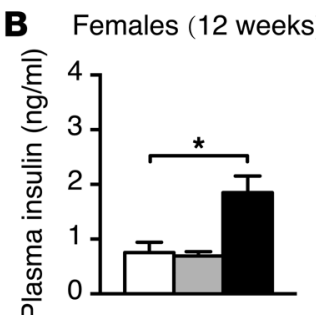
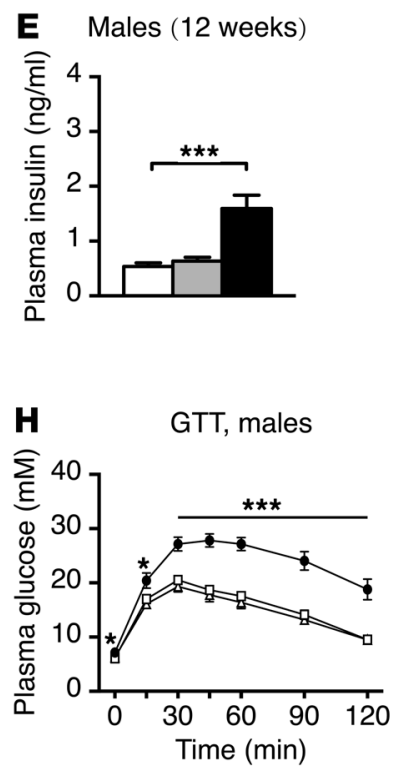

C
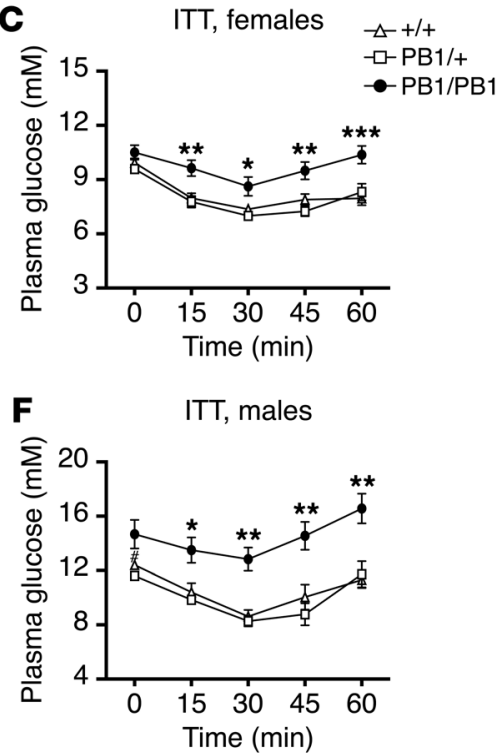

I Males (15 weeks)

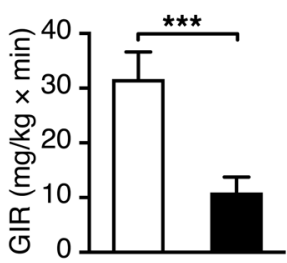

Figure 3. Disruption of Gpr45 impairs hormone and glucose homeostasis. (A and D) ELISA analysis revealed elevated plasma leptin levels in both 12-week-old female (A) and male (D) mutants. (B and E) ELISA analysis revealed elevated plasma insulin levels in both 12-week-old female (B) and male (E) mutants. At least 4 mice were used for each genotype. (C and F) Insulin tolerance test (ITT) results of 15-week-old female (C, $+/+, n=15,267.6 \pm 3.9 \mathrm{U}$ insulin; PB1/+, $n=21,273.1 \pm 4.9 \mathrm{U}$ insulin; PB1/PB1, $n=15,375.3 \pm 11.1 \mathrm{U}$ insulin) and male (F, $+/+, n=8,345.3 \pm 3.5 \mathrm{U}$ insulin; PB1/+, $n=10,350.9 \pm 3.3$ $\mathrm{U}$ insulin; PB1/PB1, $n=12,441.7 \pm 13.5 \mathrm{U}$ insulin) mice. Wild-type data were used as the statistical control. (G and $\mathbf{H})$ Glucose tolerance test (GTT) results of 12-week-old female $(\mathbf{G},+/+, n=12 ; \mathrm{PB} 1 /+, n=12$; PB1/PB1, $n=12)$ and male $(\mathbf{H},+/+, n=8 ; \mathrm{PB} 1 /+, n=8 ; \mathrm{PB} 1 / \mathrm{PB} 1, n=8)$ mice. Wild-type data were used as the statistical control. (I) Glucose infusion rate (GIR) determined by hyperinsulinemic-euglycemic clamp on 15 -week-old male mice $(n=5)$. All data are shown as the mean \pm SEM. ${ }^{*} P<0.05,{ }^{*} P<0.01$, and ${ }^{* *} P<0.001$ by Student's $t$ test; ${ }^{*} P<0.05, \mathrm{~PB} 1 /+$ versus $+/+$ in $\mathbf{F}$.

indicate that reduced physical activity contributes to decreased $\mathrm{EE}$ in obese Gpr45 mutants.

Basal metabolic rate can be measured by EE under resting phase or indicated by body temperature under normal conditions. Considering that the FVB strain in which the Gpr45 mutation was induced has fragmented and arrhythmic activity under normal light/dark cycles (46), we used body temperature as an indication of the EE of basal metabolism. Gpr45 ${ }^{P B 1 / P B 1}$ mice have rectal temperatures comparable with those of their wild-type littermates at P14 (Figure 5D). Similar results were observed when they started to have higher fat/lean ratios at the age of 3 weeks (Supplemental Figure 3, G and $\mathrm{H}$ ). However, rectal temperatures measured in 11-week-old female and male $\operatorname{Gpr} 45^{P B 1 / P B 1}$ mice were $1.4^{\circ} \mathrm{C}$ and $1.1^{\circ} \mathrm{C}$ lower than their wild-type littermates, respectively (Supplemental Figure 3, G and H), suggesting that Gpr45 mutants have a lower level of basal metabolism upon obesity.

Adaptive thermogenesis enables the animal to maintain stable body temperature in a cold environment. To evaluate whether altered adaptive thermogenesis contributes to decreased EE in Gpr45 mutants, we measured their cold-induced thermogenesis performance at P14. Deprived of food at $4^{\circ} \mathrm{C}$ for 2 hours, female and male wild-type mice had reduced rectal temperatures $\left(\right.$ by $13.7^{\circ} \mathrm{C}$ and $17.3^{\circ} \mathrm{C}$, respectively). In contrast, female and male $G p r 45^{P B 1 / P B 1}$ mice had $25.8^{\circ} \mathrm{C}$ and $25.6^{\circ} \mathrm{C}$ decreases in rectal temperatures, respectively (Figure 5D). The fact that Gpr 45 mutants are less capable of stabilizing core body temperature in acute cold exposure suggests a contribution of inefficient adaptive thermogenesis to reduced EE.

Taken together, these data indicate that disruption of Gpr45 expression decreases the rates of physical activity, basal metabolism, and adaptive thermogenesis. Therefore, obesity observed in the Gpr 45 mutants is a result of reduced EE, but not excessive energy intake.

Gpr45 regulates Pomc expression in the hypothalamus. We next asked how GPR45 affects EE in mice. A previous study reported that Gpr45 is specifically expressed in the brain (35). We found that Gpr45 is expressed not only in the brain, but also in the testis (Figure 6A). In the brain, Gpr45 mRNA was detected in the olfactory bulb, cerebral cortex, hippocampus, hypothalamus, cerebellum, and brain stem (Figure 6A), consistent with the potential neuronal role of GPR45 in the regulation of EE. 
A

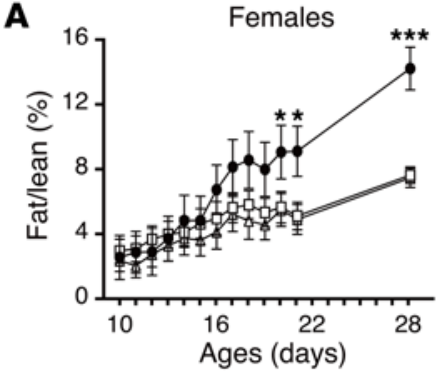

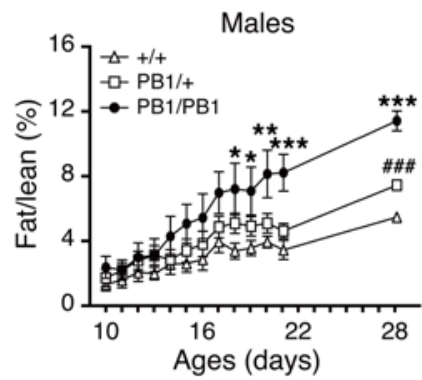

D

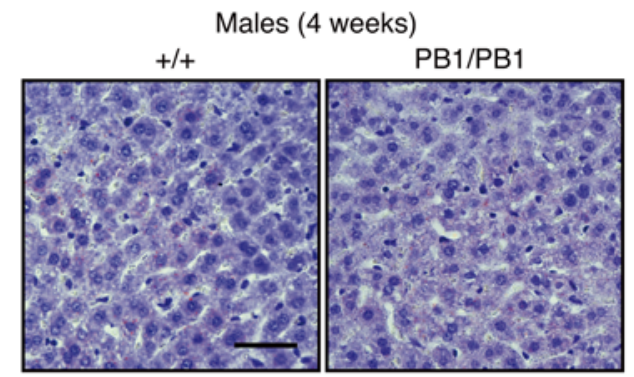

B

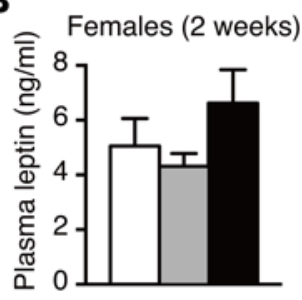

C

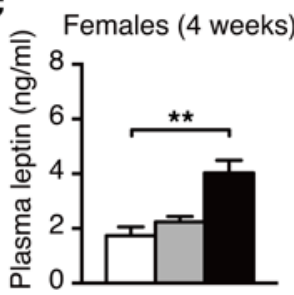

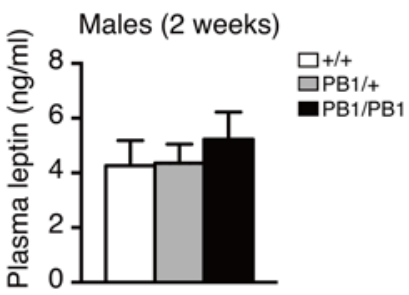

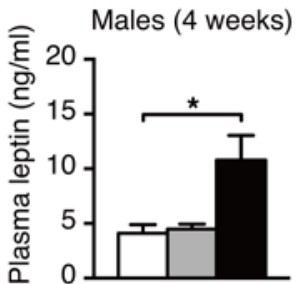

E

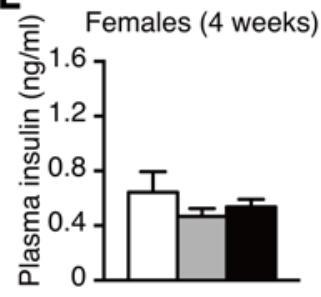

$\mathbf{F}$

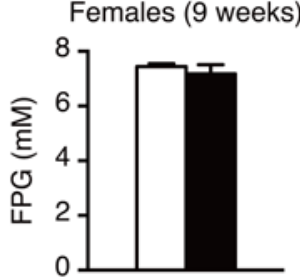

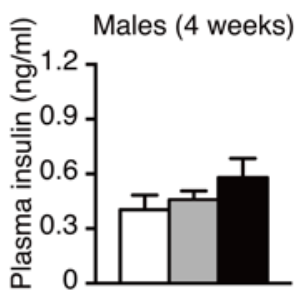

Males (9 weeks)

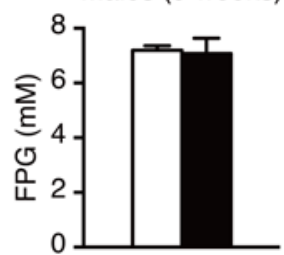

Figure 4. Obesity is the primary defect in Gpr45 mutants. (A) Daily fat/lean ratio of female $(+/+, n=9 ; \mathrm{PB} 1 /+, n=18 ; \mathrm{PB} 1 / \mathrm{PB} 1, n=8)$ and male $(+/+, n=9$; $\mathrm{PB} 1 /+, n=18$; PB1/PB1, $n=8$ ) mice revealed early-onset obesity before weaning. Data from wild-type mice were used as the control for the $P$ value calculation. (B) Bar graphs showing similar plasma leptin levels found in 2-week-old mutant and wild-type mice. At least 7 female or 6 male mice were analyzed for each genotype. (C) Bar graphs showing higher plasma leptin levels in 4-week-old mutant mice than those in wild-type littermates. Number of mice: female, +/+, 7; PB1/+, 11; PB1/PB1, 11; male +/+, 7; PB1/+, 8; PB1/PB1, 6. (D) Oil red 0 and hematoxylin staining detecting no hepatic steatosis in a 4-weekold male mouse. Scale bar: $0.05 \mathrm{~mm}$. (E) Bar graphs showing normal plasma insulin levels in 4-week-old mutant mice. Number of mice: female, $+/+, n=7$; PB1/+, $n=11$; PB1/PB1, $n=10$; male, $+/+, n=5$; PB1/,$+ n=4$; PB1/PB1, $n=6$. (F) Bar graphs showing no difference in fasted plasma glucose (FPG) levels of 9 -week-old female $(+/+, n=5 ; \mathrm{PB} 1 / \mathrm{PB} 1, n=4)$ and male $(+/+, n=5 ; \mathrm{PB} 1 / \mathrm{PB} 1, n=4)$ mice. All data are shown as the mean $\pm \mathrm{SEM}$. ${ }^{*} P<0.05,{ }^{* *} P<0.01$, and ${ }^{* * *} P<0.001$ by Student's $t$ test; ${ }^{*} \# P<0.001, \mathrm{~PB} 1 /+$ versus $+/+$ in $\mathbf{A}$.

The hypothalamus serves as the center controlling energy homeostasis. Previous studies have shown that neuropeptides involved in regulating EE, such as AGRP, NPY, POMC, CART, $\mathrm{CRH}, \mathrm{TRH}, \mathrm{BDNF}, \mathrm{MCH}$, and orexin, are all expressed in the hypothalamus (10-12). To explore if any of these molecules may mediate GPR45 regulation of EE, we analyzed the expression of these genes in the hypothalamus of P14 Gpr $45^{P B 1 / P B 1}$ mice by realtime RT-PCR. While the expression levels of most genes were not affected by the Gpr 45 mutation, Pomc transcripts were reduced by approximately $40 \%$ (Figure 6B). Western analysis confirmed a decrease in POMC protein of approximately $50 \%$ (Figure $6 \mathrm{C}$ ). POMC is expressed in Arc neurons in the hypothalamus (47). Immunofluorescent staining of sections throughout the rostralcaudal axis of Arc also revealed a significant reduction in POMCexpressing cells in P14 mutants (Supplemental Figure 4, A and B). We also analyzed if the Pomc mRNA level could be affected by Gpr45 expression in primary hypothalamic cultures. Pomc expression in cultured cells from embryonic Gpr45 ${ }^{P B 1 / P B 1}$ mutants was reduced by $84 \%$ (Figure 6D). Consistently, reducing the Gpr 45 mRNA level by $53.4 \%$ in wild-type primary cultures led to reduc- tion of Pomc mRNA by 30.7\% (Figure 6E). Taken together, these data suggest that GPR 45 promotes POMC expression.

We further examined electrophysiological activities of POMCexpressing neurons in Gpr45 mutants. As previously reported, reduced secretion of the POMC cleavage product $\alpha$-MSH was usually accompanied by reduced action potential firing (48). By current-clamp recording, we found that POMC-expressing neurons from P14 Gpr $45^{P B 1 / P B 1}$ mice had more hyperpolarized membrane potentials $(-38.4 \pm 1.6 \mathrm{mV}, P<0.01$, Figure $6, \mathrm{~F}$ and $\mathrm{G})$ and reduced spontaneous firing rates $(1.8 \pm 0.5 \mathrm{~Hz}, P=0.06$, Figure 6 , $\mathrm{F}$ and $\mathrm{H})$ than those in the wide-type littermates $(-32.4 \pm 1.3 \mathrm{mV}$ and $3.7 \pm 0.8 \mathrm{~Hz}$, respectively). These results are consistent with the previous report and suggest that the reduced firing rate may be caused by the more hyperpolarized resting membrane potential. Taken together, these data suggest that GPR45 is also involved in regulating the activity of POMC-expressing neurons.

We next explored the molecular mechanisms underlying GPR 45 regulation of Pomc, which is known to contain functional STAT3-responsive elements in its enhancer and promoter (49, 50). In the hypothalamus, Pomc transcription can be activated by 

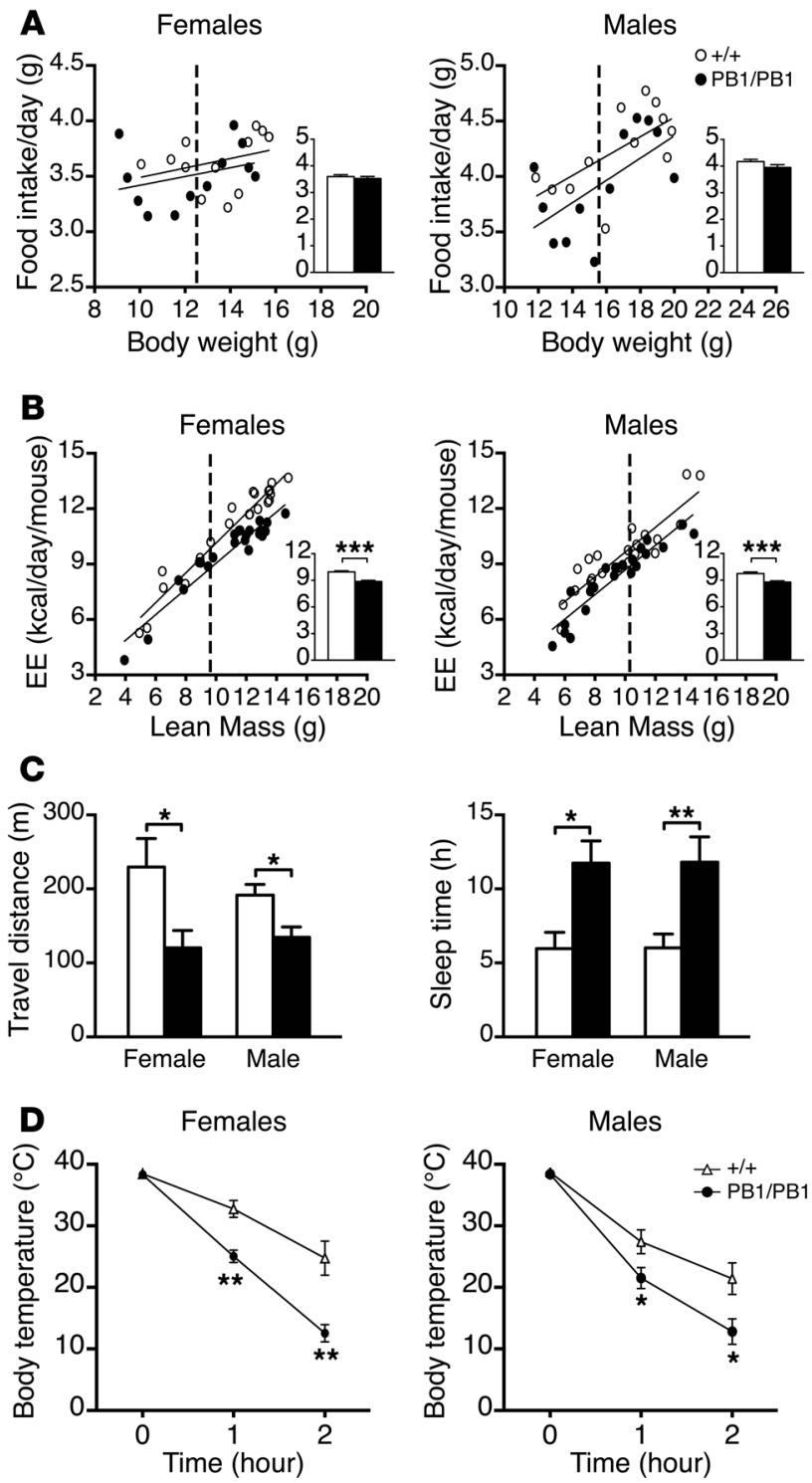

phosphorylated STAT3 $(50,51)$. Neuronal deletion of Stat3 resulted in marked reduction of POMC expression, thermal dysregulation, and obesity in mice (52). In addition, studies in cultured cells and in the hypothalamus showed that STAT3 activation depends on the phosphorylation by the protein-tyrosine kinase JAK2 (5355). We have observed decreased JAK2/STAT3 signals in Gpr45 mutants. Compared with those in wild-type littermates, JAK2 phosphorylation in cultured embryonic $G p r 45^{P B 1 / P B 1}$ hypothalamic cells was reduced by $53.2 \%$ (Figure 7A). In the hypothalamus of P14 Gpr $45^{\mathrm{PB} / \mathrm{PB} 1}$ mutants, JAK2 phosphorylation was reduced by $64.6 \%$ (Figure 7B). Phosphorylated STAT3 was also reduced by $57.9 \%$ and $40.9 \%$ in mutant embryonic and P14 hypothalamus, respectively (Figure 7, A and B). Considering that JAK2 could directly interact with several GPCRs (53-56), while Gpr45 expression has been detected in POMC neurons (57), we next tested if JAK2 and GPR 45 could physically interact. We expressed HA-tagged GPR 45 under the control of the neuron-specific enolase (NSE) promoter in mice (58). Immunoprecipitation analysis with the brain tissue of HA-GPR 45 transgenic mice revealed that GPR 45 could interact
Figure 5. Disruption of Gpr45 reduces energy expenditure. (A) ANCOVAestimated daily food intake of female $(+/+, n=8$; PB1/PB1, $n=7)$ and male $(+/+, n=9 ; \mathrm{PB} 1 / \mathrm{PB} 1, n=6)$ mice during the ages between P21 and P33. Bar graphs showing similar daily food intake in mutants estimated at a common body weight of $12.58 \mathrm{~g}$ and $15.85 \mathrm{~g}$ for female and male mice, respectively. (B) ANCOVA-estimated 24-hour total energy expenditure (EE) of female (+/+, $n=22$; PB1/PB1, $n=22)$ and male $(+/+, n=22$; PB1/PB1, $n=22$ ) mice during the ages between $\mathrm{P} 21$ and $\mathrm{P} 33$ at room temperature $\left(22^{\circ} \mathrm{C}\right)$. Bar graphs showing decreased $\mathrm{EE}$ in mutants estimated at a common lean mass of $9.76 \mathrm{~g}$ and $10.155 \mathrm{~g}$ for females and males, respectively. (C) Bar graphs showing reduced 24-hour travel distance but extended sleeping time in P21 female $(+/+, n=6$; PB1/PB1, $n=7)$ and male $(+/+$, $n=8$; PB1/PB1, $n=6$ ) mutants. $\mathrm{m}$, distance traveled in meters. (D) Body temperatures of P14 female $(+/+, n=6$; PB1/PB1, $n=6)$ and male $(+/+$, $n=22$; PB1/PB1, $n=22$ ) mice after cold exposure $\left(4^{\circ} \mathrm{C}\right)$. All data are shown as the mean \pm SEM. ${ }^{* * *} P<0.001$ by ANCOVA analysis (A and $\left.\mathbf{B}\right) .{ }^{*} P<0.05$ and ${ }^{* *} P<0.01$ by Student's $t$ test ( $C$ and $\left.\mathbf{D}\right)$.

with JAK2 (Figure 7C). An in vitro binding assay also confirmed the interaction between JAK2 and a carboxyl-terminal fragment, but not the third intracellular loop of GPR45 (Figure 7D). Furthermore, chromatin immunoprecipitation (ChIP) analysis revealed significantly reduced STAT3 recruitment to the Pomc promoter in the mutant hypothalamus (Figure 7E). Taken together, these results suggest that the JAK2/STAT3 cascade plays a significant role in mediating the regulation of Pomc transcription by GPR 45 .

Elevated POMC signal suppresses GPR 45 related obesity. To obtain further support that POMC signaling is the major downstream target of GPR 45 in preventing obesity, we performed intra-third-ventricle injection of melanotan-2 (MTII) in Gpr45 mutants. MTII is a MC3R/MC4R agonist that mimics the effect of $\alpha$-MSH to inhibit food intake and promote $\operatorname{EE}(14,17,59)$. MTII treatment caused similar amounts of food intake reduction in both mutant and wild-type mice (Figure 7G). Compared with those of saline-treated control mice, cumulative food intake after 6-day MTII injection decreased $12 \%$ and $14.8 \%$ in wild-type and mutant animals, respectively. In contrast, daily injection of MTII led to substantially more weight loss in $G p r 45^{P B 1 / P B 1}$ mice than in wild-type animals (Figure 7F). Three-day MTII treatment was sufficient to reduce the body weight of $G p r 45^{P B 1 / P B 1}$ mice to the level of saline-treated wild-type littermates. After 6 days of injection, MTII-treated mutant mice lost $11.7 \%$ of their original body weight, while MTII-treated wild-type animals only lost $1.2 \%$. Compared with those of saline-treated mutants, average weights of perigonadal, retroperitoneal, and interscapular fat mass in MTII-treated mutants were $52.6 \%, 63.3 \%$, and $50.3 \%$ lower after 6 days of MTII injection, respectively. In wild-type mice, perigonadal and retroperitoneal fat mass in the treated group were $44.5 \%$ and $49.8 \%$ lower than those in the control group, respectively. Interscapular fat mass, on the other hand, was $0.5 \%$ higher after MTII administration (Figure 7H). The more sensitive response of $G p r 45^{P B 1 / P B I}$ mice to a drug activating POMC signaling suggests that GPR45 likely regulates metabolism through this pathway.

\section{Discussion}

We observed obesity, hepatic steatosis, and hyperglycemia in Gpr45 mutant mice. Initially identified as the mammalian orthologue of a putative Xenopus lysophosphatidic acid (LPA) receptor, GPR45 was later recognized as an orphan GPCR with no response 
A
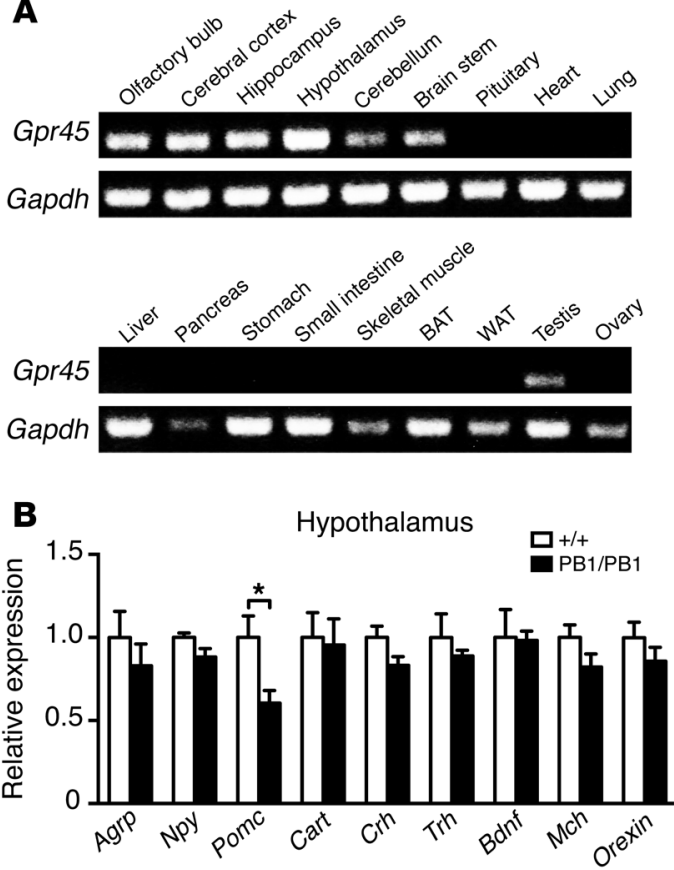

C Hypothalamus
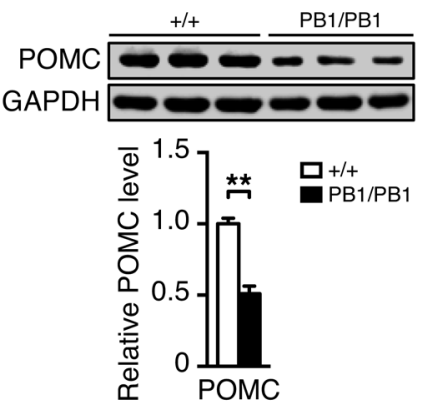

D

Primary hypothalamic cultures

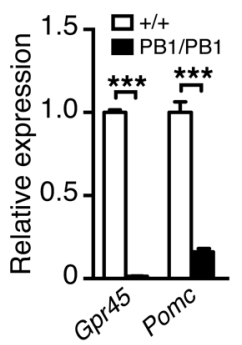

$\mathbf{F}$

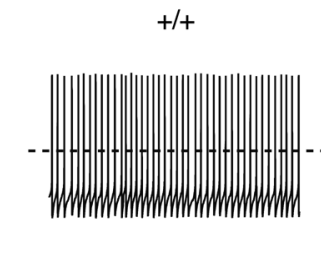

G

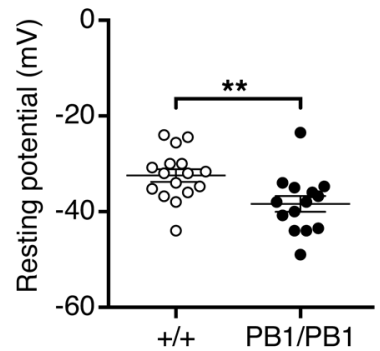

E

Primary hypothalamic cultures

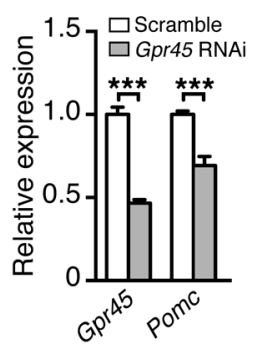

$\mathrm{PB} 1 / \mathrm{PB} 1$

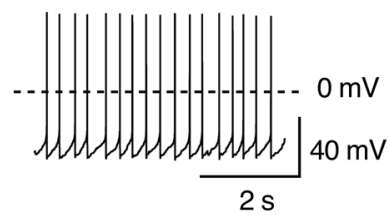

H

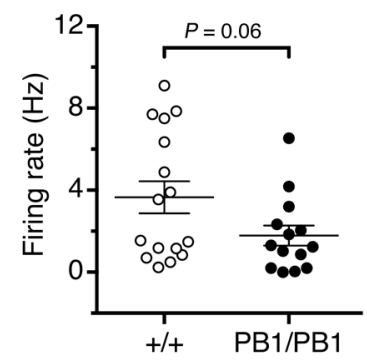

Figure 6. Disruption of Gpr45 decreases hypothalamic POMC expression and spontaneous firing rate of POMC neurons. (A) Results of RT-PCR analysis showing the presence of Gpr45 mRNA in neuronal tissues and the testes of adult mice. (B) Real-time RT-PCR analysis revealed decreased Pomc, but similar expression of other hypothalamic neuropeptides in P14 mice ( $n \geq 4$ for each genotype). Expression of Gapdh serves as the internal control to calculate relative expression levels. (C) Western blots show decreased POMC levels in hypothalami of P14 mice ( $n=3$ for each genotype). A quantitative comparison is listed below. Expression of GAPDH serves as the internal control to calculate relative expression levels. (D) Bar graphs showing significantly reduced Gpr45 and Pomc expression in primary hypothalamic cells from the mutants. Summarized real-time RT-PCR results from 3 independent wells are shown, with Gapdh serving as the internal control. (E) Knockdown of Gpr45 in primary hypothalamic cells caused down regulation of Pomc. Real-time RT-PCR data were collected with the same procedure in (D), except for the use of 5 wells in each group. (F) Representative recordings of resting membrane potential from wild-type ( $n=16$ from 6 mice) and PB1/PB1 ( $n=14$ from 3 mice) animals. ( $G$ and $\mathbf{H})$ Statistics for resting membrane potential (G) and spontaneous firing rate $(\mathbf{H})$ in these recordings. All data are shown as the mean \pm SEM. ${ }^{*} P<0.05,{ }^{* *} P<0.01$, and ${ }^{* * *} P<0.001$ by Student's $t$ test.

to LPA $(35,60)$. Gpr45 is expressed in the CNS of both male and female mice. Disruption of Gpr 45 results in reduced POMC expression and decreased EE. Intraventricular injection of MTII, an analog of POMC-derived hormones, effectively reverses the obesity caused by Gpr45 mutations. These results revealed an important role of GPR45 in energy homeostasis. Due to their functional diversity and membrane-bound features (61), GPCRs are considered to be the most attractive drug target candidates, as more than a third of commercial drugs are generated by modifying the functions of different GPCRs (62). Our results suggest that developing a GPR45-specific agonist may help the treatment of obesity.

Altered lean mass is not uncommon in obese mice. Although partial reduction of hypothalamic Pomc transcription in the FVB strain resulted in obesity without affecting lean mass (16), most Pomc mutants generated on 129 and/or C57/BL6 backgrounds showed both obesity and increased lean mass $(19,63)$. Since lean mass is the principal site of glucose disposal, caution is warranted when interpreting glucose metabolic data in these mutants, especially if the assays were performed based on the body weight (64). Gpr $45^{P B 1 / P B 1}$ mice develop obesity along with reduced lean mass (Figure 2H). Thus, dosing insulin based on the body weight in ITT tends to result in an underestimation of insulin resistance (Figure 3, C and F), while dosing glucose based on the body weight in GTT tends to result in an overestimation of glucose intolerance (Figure 3, G and H). Therefore, we further tested insulin resistance by hyperinsulinemic-euglycemic clamp, the gold standard for assess- 
A

Primary hypothalamic cultures
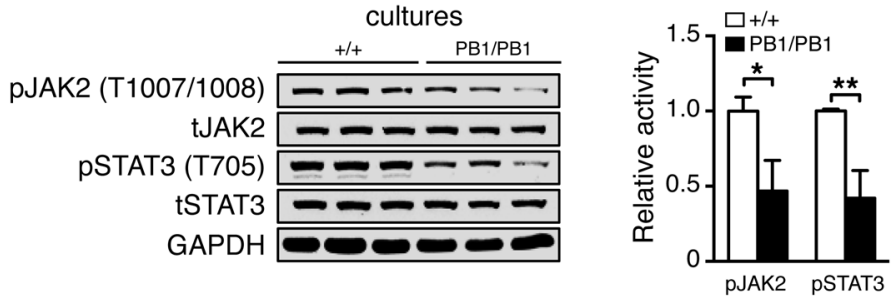

B
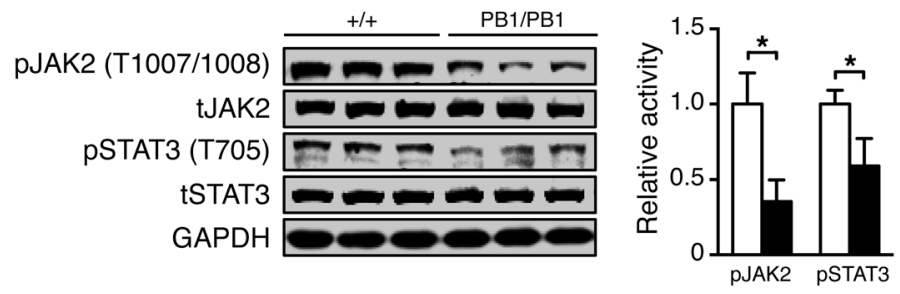

C

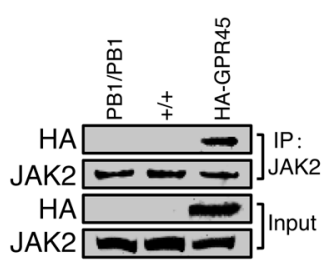

D

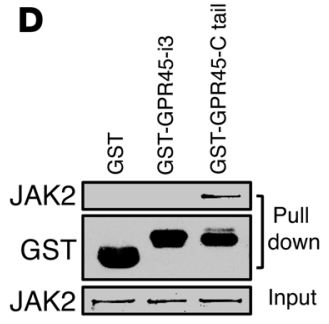

E

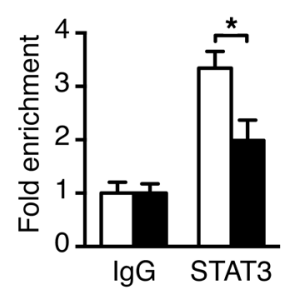

$\mathbf{F}$

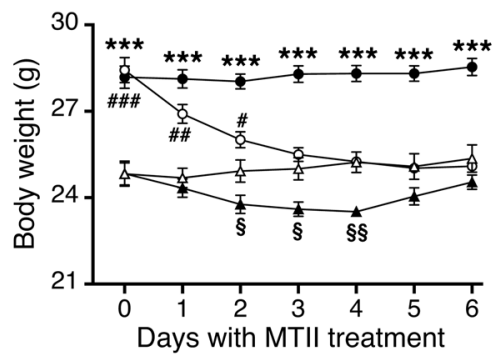

G

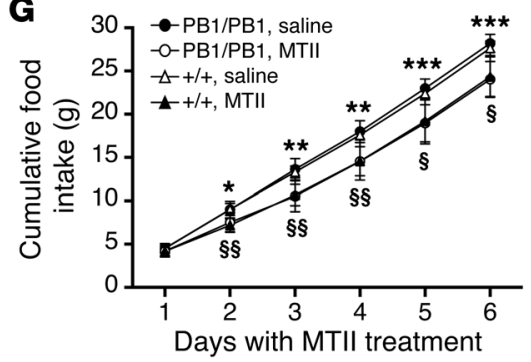

Figure 7. GPR45 regulates POMC for its role in energy expenditure. (A and B) Western blots shows decreased phosphorylation of JAK2 and STAT3 in primary mutant hypothalamic cells (A) and hypothalami of P14 mutants (B). Quantitative comparisons are shown on the right, with total JAK2 or STAT3 protein as the internal control. $n=3$ for each genotype. (C and $\mathbf{D})$ Physical interaction between JAK2 and GPR45. Immunoprecipitation (C) by anti-JAK2 antibody showing that GPR45 interacts with JAK2 in the brain of adult HA-GPR45 transgenic mice. Anti-HA antibody was used to recognize HA-tagged GPR45. In vitro binding assay (D) shows that JAK2 interacts with the carboxyl terminus (GPR45-C tail), but not the third intracellular loop of GPR45 (GPR45-i3). Anti-GST antibody was used to recognize GST-tagged GPR45 fragments. (E) Chromatin immunoprecipitation by anti-STAT3 antibody revealed reduced STAT3 recruitment to the Pomc promoter in hypothalami of P14 mutants $(n=4)$. (F and $\mathbf{H})$ Intra-third-ventrical administration of MTII reduced both bodyweight (F) and fat mass (H) in adult male mutants. gWAT, perigonadal fat; rWAT, retroperitoneal fat; BAT, interscapular brown fat. (G) MTII treatment reduced food intake in Gpr45 mutants and their wild-type littermates. Five mice were used for each group. Data from saline-treated wild-type mice serve as the baseline for statistics. All data are shown as the mean \pm SEM. ${ }^{*} P<0.05,{ }^{* *} P<0.01$, and ${ }^{* * *} P<0.001$ by Student's $t$ test; ${ }^{*} P<0.05$, $\# P<0.01$, and $\# \# P<0.001$, MTII-treated PB1/PB1 vs. saline-treated $+/+; \$ P<0.05$ and $\$ \$ P<0.01$, MTII-treated $+/+$ vs. saline-treated $+/+$ in $\mathbf{F}$ and $\mathbf{G}$.

ing insulin sensitivity (Figure 3I). The blood glucose data were also normalized by lean mass to confirm the conclusion. Since all other metabolic defects developed after the initiation of obesity in Gpr 45 mutants, we conclude that obesity is the primary physiological defect upon the disruption of Gpr45.

Both decreased EE and obesity found in the Gpr45 loss-offunction mutants have also been reported in mice carrying hypothalamic deletion of Pomc $(18,63)$. However, the Gpr45 mutants do not develop hyperphagia, which is usually observed in Pomc mutants. This difference could result from dosage variation of Pomc, as loss of Gpr45 caused only partial reduction of POMC expression in the hypothalamus. Consistent with this explanation, partial reduction of hypothalamic Pomc transcription has been shown to cause obesity, but not hyperphagia (16). Alternatively, the feeding difference between Gpr45 and Pomc mutants may reflect the heterogeneity of the regulation and function of POMC signaling in the hypothalamus. In fact, functionally varied POMC neurons could be further distinguished with different markers. RNA in situ hybridization has shown that approximately $30 \%$ of POMC neurons express the insulin receptor, while the ratio of LEPR-positive POMC neurons varied from $32.8 \%$ to $89.6 \%$ (65, 66). LEPR-positive POMC neurons could not be depolarized by $\mathrm{mCPP}$, a serotonin $2 \mathrm{C}$ receptor agonist, while some LEPR-negative POMC neurons were depolarized (67). GPR45 may only function in a subpopulation of POMC neurons, so that partial reduction of hypothalamic POMC may result from severe impairment of POMC expression in these cells, or even the loss of these neurons. Consistent with this assumption, we observed a similar extent of decrease in food intake after MTII treatment in Gpr45 mutants and the wild-type mice, which suggests that MTII-related food intake regulation is unaffected in the mutants. In the same experiment, more severe weight loss in Gpr 45 mutants also implies that a different body weight-controlling mechanism, likely EE regulation, is imbalanced. Although we provided multiple lines of evi- 
dence supporting a critical role for POMC in mediating GPR45dependent regulation of energy homeostasis, our data do not lead to the conclusion that POMC is the only downstream target.

Disruption of GPR45 does not affect food intake. This result does not exclude the possibility that food uptake may still impact GRP45 function. A transcriptomic study has reported identical Gpr45 expression in both POMC and AGRP neurons, which was not affected by altered food intake (57). This result also does not exclude the possibility that food intake affects GPR45 function through a mechanism other than regulation of expression. Given the broad expression of Gpr45 in neuronal tissues (Figure 6A), understanding the relationship between food intake and GPR45 function, as well as other GPR45-involved processes, would be interesting directions for future studies.

In addition to Gpr45, we identified 4 genes that were not known to be involved in obesity from the pilot screen (Figure 1, $\mathrm{C}$ and D). The gene encoding ephrin receptor B1 (Ephb1) is critical for axon guidance and synaptogenesis in the brain (68). The Ubr3 gene encodes a ubiquitin protein ligase E3 component that has been shown to be necessary for olfaction of neonatal mice (69). The Fbxw2 gene encodes an F-box and WD-40 domain protein and has been shown to positively regulate ubiquitination of hGCM1, a zinc-containing transcription factor involved in placental and parathyroid development $(70,71)$. The protein encoded by Eepd1 is known to interact with CIA1 (cytosolic iron-sulfur protein assembly component 1 ) and potentially contribute to the maturation of $\mathrm{Fe} / \mathrm{S}$ proteins (72). These genes are involved in different biological processes, showing the complexity of molecular mechanisms underlying obesity.

In recent years, a number of large GWAS efforts have revealed strong associations between dozens of candidate genes with obesity. However, such studies typically provide little insight into the disease mechanisms. Furthermore, the genetic risk predicted by GWASs is far less than estimated heritability in obesity. We applied an unbiased phenotypic screen strategy in a collection of mouse mutants. Compared with human populations, inbred mice provide a purified genetic background that allows easier recognition of phenotypic changes. Once identified, mutant mice can immediately be used for mechanistic studies. Thus, genetic screens of mouse mutants provide an efficient complementary approach to studying the genetic regulation of obesity. With this approach, we successfully identified 5 genes that were not previously known to be involved in obesity.

During the primary screen, we identified 14 overweight mutants from 408 lines. In the subsequent round of analysis, 7 obese mutants were identified. Thus, the overall positive ratio is approximately $1.7 \%$ for the screen. This is likely an underestimation of the loci involved in obesity for the following reasons. First, $P B$ insertions often cause loss-of-function mutations, so that genes stimulating fat deposition are expected to be underrepresented in the primary screen for overweight mutants. Second, the current pipeline relies on body weight alteration to find obesity loci, so that mutations only affecting body composition would not be recognized. In fact, body weight may not be the best screen criterion, since mutations of metabolic regulators may not change body weight. For example, $M c 3 r$ knockout mice display significant obesity but normal body weight (20). In addition, our pilot screen did reveal imperfect repeatability of body weight in Fshr and Eepd1 mutants. Implementation of more sensitive phenotyping techniques, such as body composition analysis, in the primary screen should help to identify more genes involved in obesity.

\section{Methods}

Mice. All mutants were generated on the FVB/NJ background and maintained on 12/12-hour light/dark cycles. Mapping information of PB insertions in Gpr45 and other genes can be found in the PBmice database (idm.fudan.edu.cn/PBmice).

General physiology and behavior tests. Mice were weighed and placed in a miniSpec NMR instrument (Bruker) to measure the quantity and ratio of body fat, lean, and liquid compositions. Before plasma hormone tests, a fasting period of 4 or 6 hours was applied to 4- or 12 -week-old mice, respectively. Blood samples were then collected form the orbital sinus and analyzed with Mouse Leptin and Insulin Ultrasensitive ELISA Kits (Crystal Chem, Inc.). For fecal energy tests, fecal pellets were collected for 24 hours from individually housed mice, dried at $60^{\circ} \mathrm{C}$ for 3 days, and burned in a bomb calorimeter (Heitersheim). Benzoic acid (BHD Limited) was used as a thermochemical standard. To score travel distance and sleeping time, mice were acclimated in a HomeCageScan system (Clever Sys, Inc.) for 24 hours before another 24-hour recording. Sleep is recognized as a period of inactivity for more than 30 seconds (73). The SHIRPA analysis was performed according to a previously described protocol (74). Investigators were blinded to genotypes during assessments with the help of well-trained technicians.

Metabolic assays. For GTTs, 12-week-old mice were fasted for 16 hours before receiving intraperitoneal injection of $20 \%$ glucose saline solution ( $2 \mathrm{~g}$ glucose per $\mathrm{kg}$ body weight). Tail vein blood was sampled at $0,15,30,45,60,90$, and 120 minutes after injection, and analyzed with a Onetouch Ultra blood glucose monitoring system (LifeScan). For the ITTs, 15-week-old free-fed mice received an intraperitoneal injection of insulin (Humulin, Lilly) (0.75 U/kg body weight), then had tail vein blood glucose levels measured $0,15,30$, 45 , and 60 minutes later. For the hyperinsulinemic-euglycemic clamp experiments, indwelling catheters were implanted into the right internal jugular vein of sampled mice. After 5 days of recovery, mice were fasted overnight and then studied in a restrainer. The clamp was initiated with continuous infusion of human insulin $(18 \mathrm{mU} /$ $\mathrm{kg} \cdot \mathrm{min})$ for 120 minutes. Physiological blood glucose level (6.7-7.2 $\mathrm{mM}$ ) was maintained by measuring blood glucose every 10 minutes and adjusting the infusion rate of $20 \%$ glucose. The glucose infusion rate data were collected after a 90 -minute equilibration period. To measure total $\mathrm{EE}$ and respiratory quotient, an indirect calorimetric system from Promethion was used to house animals supplied with the standard chow diet at $22^{\circ} \mathrm{C}$. Mice were recorded for 48 hours after a 24-hour cage acclimation. To measure body temperature alterations in the cold environment, P14 mice were individually housed at $4^{\circ} \mathrm{C}$ with no food but plenty of water. Rectal temperatures were recorded with a thermoprobe at 0,1 , and 2 hours after cold exposure. The mice were euthanized immediately at the end of the test.

Histology and immunohistochemistry. To examine hepatic steatosis, $10-\mu \mathrm{m}$ frozen liver sections were prepared from $4 \%$ paraformaldehyde-fixed (PFA-fixed) tissues and stained with hematoxylin and $0.5 \%$ Oil red O. To check gene expression in the hypothalamus, brains from transcardially perfused mice were postfixed overnight 
with $4 \%$ PFA, cryoprotected by incubating in $30 \%$ sucrose at $4^{\circ} \mathrm{C}$ for 2 days, and sectioned at a thickness of $25 \mu \mathrm{m}$ for immunostaining. Rabbit anti-POMC (Phoenix; catalog H02930; 1:800) and donkey anti-rabbit Alexa Fluor 488 (Invitrogen; catalog A-21206; 1:1,000) antibodies were used for POMC. Images were collected using a Zeiss LSM510 microscope.

PCR and Western blot. Total RNA was extracted with TRIzol (Invitrogen) and reverse transcribed (Takara) for RT-PCR. Realtime PCR was performed using Brilliant QPCR Master Mix (Agilent) following the manufacturer's standard-curve method. Relative expression of each target gene was calculated by comparison to the expression of mouse Gapdh, and presented as relative to the average ratio of wild-type littermates, which is arbitrarily defined as 1 . Four to six animals were examined for each genotype. Without further notification, groups with equal numbers of samples were scored in each experiment.

For Western blot, cells were homogenized in ice-cold RIPA buffer and centrifuged at $15,000 \mathrm{~g}$. The supernatant was resolved by $10 \%$ SDS-PAGE, electrotransferred to a PVDF membrane, and probed with the following antibodies: goat anti-POMC (Abcam; catalog ab32893; 1:500), rabbit anti-pJAK2-Tyr ${ }^{1007 / 1008}$ (Cell Signaling Technology; cata$\log 3776 ; 1: 1,000$ ), rabbit anti-JAK2 (Cell Signaling Technology; catalog 3230; 1:1,000), rabbit anti-pSTAT3-Tyr ${ }^{705}$ (Cell Signaling Technology; catalog 9145; 1:1,000), rabbit anti-STAT3 (ABclonal; catalog A1192; 1:500), mouse anti-HA (Covance; catalog MMS-101P; 1:2,000), rabbit anti-GST (Sangon Biotech; catalog AB10271; 1:4,000), goat antirabbit IgG-HRP (Santa Cruz Biotechnology; catalog sc2004; 1:4,000), bovine anti-goat IgG-HRP (Santa Cruz Biotechnology; catalog sc2352; 1:4,000) and goat anti-mouse IgG-HRP (Santa Cruz Biotechnology; catalog sc2005; 1:4,000) antibodies. Blotting images were acquired with a Tanon-5200 imaging system. Densitometric quantification of the target protein was determined by Image J (NIH) and compared with the internal control to determine the relative expression value. The average relative expression value of each genotype is presented as relative to that of the wild-type littermates, which is arbitrarily defined as 1 . The internal controls used for POMC, pJAK2, and pSTAT3 were GAPDH, JAK2, and STAT3, respectively. The representative blots were selected from 3 repeated experiments, with 3 mice of each genotype in each experiment.

cDNA cloning and plasmid construction. To clone mouse Gpr45, total brain RNA was reverse transcribed (Takara) for RT-PCR according to the manufacturer's protocol. Full-length cDNAs were cloned into pGEM-T vector and subcloned into the EcoRI site of the pcDNA4.0HA vector. An $N h e \mathrm{I} / E c o R V$ fragment was then inserted into the HindIII site of pNSE to generate the HA-GPR45 transgenic mice (75).

To clone the GST fusion proteins containing the third intracellular loop (aa 220-265; GST-GPR45-i3) or the carboxyl terminal (aa 328-373; GST-GPR45-C tail) of GPR45, flag-tagged GPR45 fragments from $\mathrm{pNTAP}$ were subcloned into pGEX-4T1.

To clone His-fused JAK2, the full-length mouse JAK2 cDNA (a gift from Nan Li, The Second Military Medical University, Shanghai, China) was cloned with a double NotI/BamHI digestion into pET32a.

Coimmunoprecipitation and in vitro binding assay. For coimmunoprecipitation analysis, protein was prepared from mouse brains with TNP buffer (50 mM Tris- $\mathrm{HCl}$ [pH 7.4], $150 \mathrm{mM} \mathrm{NaCl}$, and 1\% NP-40), Protein A/G Agarose (Santa Cruz Biotechnology; catalog sc-2003), and rabbit anti-JAK2 antibody according to the manufacturer's protocol.
For in vitro binding assays, pure GST, recombinant GST-GPR45i3, GST-GPR45-C tail, and His-JAK2 proteins were expressed in Rossetta bacteria, purified by Glutathione Sepharose (GE Healthcare) or Ni-NTA (QIAGEN) resin according to the manufacturer's protocol. Purified proteins were incubated together at $4^{\circ} \mathrm{C}$ for 1.5 hours, PBS washed, and analyzed by Western blot.

ChIP assay. ChIP assays were performed using a SimpleCHIP Plus Enzymatic Choromatin IP Kit (Agarose beads) following the manufacturer's instructions (Cell Signaling Technology; catalog 9004). Crosslinked protein-DNA complexes were precipitated by incubating with rabbit anti-STAT3 (Cell Signaling Technology; catalog 4904; 1:50) or rabbit IgG (negative control) overnight and then with Protein G Agarose beads for 2 hours. The purified DNA fragments including STAT3binding elements were quantitatively analyzed by real-time PCR with primers against the Pomc promoter following the standard-curve method (Supplemental Table 3) (76). The standard curves were created by serial dilution of $2 \%$ input chromatin DNA. The values of chromatin DNA precipitated by STAT3 antibody were normalized to those precipitated by normal rabbit IgG, which is arbitrarily defined as 1 .

Intra-third-ventrical infusion. A cannula with an obturator stylet inside (RWD Life Science Co., LTD) was implanted into the third ventricle of 8-week-old male mice as described (77). After 1 week of recovery, mice received daily intraventricle injection of $1 \mu \mathrm{g}$ MTII 1 hour before the dark phase. Food was weighed every day 1 hour before MTII administration. At the end of the study, $2 \mu$ methylene blue was infused into the animal to confirm the correct position of drug administration.

Primary hypothalamic neuron culture. Fetal hypothalami were isolated between embryonic days 16.5 and 18.5 , minced in ice-cold $1 \times$ HBSS, and digested in $0.125 \%$ trypsin-EDTA at $37^{\circ} \mathrm{C}$ for $20-30$ minutes. Tissue blocks were gently pipetted in DMEM with $10 \%$ fetal bovine serum until no clumps remained. Suspended cells were filtered through $70-\mu \mathrm{m}$ cell strainers (Corning), incubated in poly-D-lysinecoated (Sigma-Aldrich) wells for 4 hours, and cultured in neurobasal media (Invitrogen) containing 2\% B27 (Invitrogen) and $2 \mathrm{mM} \mathrm{L-glu-}$ tamine for 7 days. Finally, cells were transfected with siRNA by Lipofectamine RNAiMAX (Invitrogen) and analyzed 72 hours later.

Electrophysiology. Whole-cell patch clamp recordings of neurons from P14 male mice were performed as previously described (78, 79). Briefly, pups were decapitated and blocks of tissue containing the arcuate nucleus were placed in artificial cerebrospinal fluid solution (125 mM NaCl, $25 \mathrm{mM} \mathrm{NaHCO}_{3}, 3 \mathrm{mM}$ myo-inositol, $2 \mathrm{mM} \mathrm{Na}-$ pyruvate, $2.5 \mathrm{mM} \mathrm{KCl}, 1.25 \mathrm{mM} \mathrm{NaH}_{2} \mathrm{PO}_{4}, 0.4 \mathrm{mM}$ ascorbic acid, 25 $\mathrm{mM}$ glucose, $3 \mathrm{mM} \mathrm{MgCl}_{2}$, and $0.05 \mathrm{mM} \mathrm{CaCl}_{2}$ ). Brain slices of 250 $\mu \mathrm{m}$ thickness were prepared using a Leica VT 1200 s vibratome and recovered at $37^{\circ} \mathrm{C}$ with $95 \% \mathrm{O}_{2}$ and $5 \% \mathrm{CO}_{2}$ for 60 minutes before experiments. Electrophysiological recordings were made at room temperature $\left(22^{\circ} \mathrm{C}-24^{\circ} \mathrm{C}\right)$. Current-clamp recordings of action potentials were made with an EPC 10 amplifier using pipettes (2.5-3.5 $\mathrm{M} \Omega$ ) containing $125 \mathrm{mM}$ K-gluconate, $20 \mathrm{mM} \mathrm{KCl,} 4 \mathrm{mM}$ Mg-ATP, $10 \mathrm{mM} \mathrm{Na}_{2}$-phosphocreatine, $0.3 \mathrm{mM} \mathrm{GTP}, 10 \mathrm{mM}$ HEPES, and 0.5 mM EGTA ( $\mathrm{pH} 7.2$, adjusted with $\mathrm{KOH}$ ). The series resistance was maintained at lower than $10 \mathrm{M} \Omega$ and the holding current at $-70 \mathrm{mV}$ was smaller than $100 \mathrm{pA}$. After recording, the content of the neuron was aspirated into the recording pipette and expelled into a test tube containing $9 \mu$ l Single Cell Lysis Solution (Ambion, catalog 4458235) and reverse transcribed by SuperScript III RT transcriptase (Invitrogen, catalog 11754-050) following the manufacturer's instructions. 
Nested PCRs were then performed to confirm the expression of Pomc (Supplemental Table 3).

Statistics. Food intake and EE data were analyzed by ANCOVA followed by 2-tailed Student's $t$ test as previously described (80). Body weight and lean mass were employed as a covariant for food intake and EE, respectively. A gradient for each group was created and all individual data points slid along imaginary parallel lines until they all grouped together at the average covariant. The intercepts were then calculated and compared between 2 genotypes. Real-time EE data were analyzed by 2-way ANOVA. SHIRPA data were analyzed by a nonparametric Mann-Whitney $U$ test. Other data were compared by unpaired 2-tailed Student's $t$ test, and shown as the mean \pm SEM. In the primary screen, body weights of homozygous mutants were compared with those of age- and sex-matched heterozygous mice in the control group. The control group consisted of 40 heterozygous animals hitting different genes, all examined no later than the latest test date of the homozygotes. Overweight mutants were identified if $P$ was less than 0.01. Starting with the secondary screen, all parameters were compared between homozygous mice and their littermates with at least 3 mice in each group. A $P$ value less than 0.05 was considered statistically significant.

Study approval. Mice were treated with protocols approved by the Animal Care and Use Committee of the Institute of Developmental Biology and Molecular Medicine, Fudan University.

\section{Author contributions}

JC performed experiments, analyzed data, and wrote the manuscript. TX, MH, and YZ designed and supervised the screen of $\mathrm{PB}$ mutants. YD, SC, XZ, and YZ participated in the screen. TL, LS, and
SZ helped analyze the screen data. XW conceived of and designed the studies, supervised the work, and wrote the manuscript. YW and LX performed the electrophysiology studies. MZ and WJ performed the hyperinsulinemic-euglycemic clamp analysis.

\section{Acknowledgments}

We thank Nan Li for providing Jak2 cDNA; Hongbo Yu, Xiaowei Dong, Zhong Li, and Wenjin Zhu for their help with intra-thirdventrical injections; Linyin Feng and Xiaoyu Yang for their help with primary hypothalamic cultures; Yan Liu and Lei Tian for their help with fecal energy analysis; Bin Guo and Yanling Yang for their help with transgenic mice; Xiaorong Huang, He Tan, Yanyan Nie, Liya Yang, Ying Yao, Yanfeng Tan, Longcheng Xu, Yueying Chen, and Yanqian Xia for their help with metabolic phenotyping; Boying Tan for animal care; Beibei Ying, Wufan Tao, Yufang Zheng, Kejing Deng, and other IDM members for valuable discussions. This work was supported in part by grants from the Chinese Hi-tech Research and Development Project (863) (grants 2014AA021104 and 2012AA022401), Natural Science Foundation of China (NSFC) (grants 81570756 and 81170789), and Shanghai Municipal Science and Technology Commission (STCSM) (grants 15XD1500500 and 12431900100).

Address correspondence to: Xiaohui Wu, Room 602, Yifu Building of Science and Technology, 220 Handan Road, Shanghai 200433, China. Phone: 86.21.6564.3718; E-mail: xiaohui_wu@fudan. edu.cn. Or to: Tian Xu, Room 236C, Boyer Center for Molecular Medicine, 295 Congress Avenue, New Haven, Connecticut 06519, USA. Phone: 203.737.2623; E-mail: tian.xu@yale.edu.
1. Flegal KM, Carroll MD, Kit BK, Ogden CL. Prevalence of obesity and trends in the distribution of body mass index among US adults, 1999-2010. JAMA. 2012;307(5):491-497.

2. Ogden CL, Carroll MD, Kit BK, Flegal KM. Prevalence of obesity and trends in body mass index among US children and adolescents, 1999-2010. JAMA. 2012;307(5):483-490.

3. Li S, Loos RJ. Progress in the genetics of common obesity: size matters. Curr Opin Lipidol. 2008;19(2):113-121.

4. Huszar D, et al. Targeted disruption of the melanocortin-4 receptor results in obesity in mice. Cell. 1997;88(1):131-141.

5. Calton MA, et al. Association of functionally significant Melanocortin-4 but not Melanocortin-3 receptor mutations with severe adult obesity in a large North American case-control study. Hum Mol Genet. 2009;18(6):1140-1147.

6. Hainerová I, et al. Melanocortin 4 receptor mutations in obese Czech children: studies of prevalence, phenotype development, weight reduction response, and functional analysis. JClin Endocrinol Metab. 2007;92(9):3689-3696.

7. Hinney A, et al. Prevalence, spectrum, and functional characterization of melanocortin- 4 receptor gene mutations in a representative populationbased sample and obese adults from Germany. JClin Endocrinol Metab. 2006;91(5):1761-1769.

8. Willer CJ, et al. Six new loci associated with body mass index highlight a neuronal influ- ence on body weight regulation. Nat Genet. 2009;41(1):25-34.

9. Thorleifsson G, et al. Genome-wide association yields new sequence variants at seven loci that associate with measures of obesity. Nat Genet. 2009;41(1):18-24.

10. Flier JS. Obesity wars: molecular progress confronts an expanding epidemic. Cell. 2004;116(2):337-350.

11. Schwartz MW, Woods SC, Porte D, Seeley RJ, Baskin DG. Central nervous system control of food intake. Nature. 2000;404(6778):661-671

12. Parker JA, Bloom SR. Hypothalamic neuropeptides and the regulation of appetite. Neuropharmacology. 2012;63(1):18-30.

13. Pritchard LE, White A. Neuropeptide processing and its impact on melanocortin pathways. Endocrinology. 2007;148(9):4201-4207.

14. Pierroz DD, Ziotopoulou M, Ungsunan L, Moschos S, Flier JS, Mantzoros CS. Effects of acute and chronic administration of the melanocortin agonist MTII in mice with diet-induced obesity. Diabetes. 2002;51(5):1337-1345.

15. Thornton JE, Cheung CC, Clifton DK, Steiner RA. Regulation of hypothalamic proopiomelanocortin mRNA by leptin in ob/ob mice. Endocrinology. 1997;138(11):5063-5066.

16. Balthasar $\mathrm{N}$, et al. Leptin receptor signaling in POMC neurons is required for normal body weight homeostasis. Neuron. 2004;42(6):983-991.

17. Yaswen L, Diehl N, Brennan MB, Hochge- schwender U. Obesity in the mouse model of pro-opiomelanocortin deficiency responds to peripheral melanocortin. Nat Med. 1999;5(9):1066-1070.

18. Zhan C, et al. Acute and long-term suppression of feeding behavior by POMC neurons in the brainstem and hypothalamus, respectively. J Neurosci. 2013;33(8):3624-3632.

19. Challis BG, et al. Mice lacking pro-opiomelanocortin are sensitive to high-fat feeding but respond normally to the acute anorectic effects of peptide-YY(3-36). Proc Natl Acad Sci U S A. 2004;101(13):4695-4700.

20. Chen AS, et al. Inactivation of the mouse melanocortin-3 receptor results in increased fat mass and reduced lean body mass. Nat Genet. 2000;26(1):97-102.

21. Hummel KP, Dickie MM, Coleman DL. Diabetes, a new mutation in the mouse. Science. 1966;153(3740):1127-1128.

22. Ingalls AM, Dickie MM, Snell GD. Obese, a new mutation in the house mouse. J Hered. 1950;41(12):317-318.

23. Mencarelli M, et al. A novel missense mutation in the signal peptide of the human POMC gene: a possible additional link between early-onset type 2 diabetes and obesity. Eur J Hum Genet. 2012;20(12):1290-1294.

24. Cieslak J, Majewska KA, Tomaszewska A, Skowronska B, Fichna P, Switonski M. Common polymorphism (81Val $>$ Ile) and rare mutations 
(257Arg $>$ Ser and 335Ile $>$ Ser) of the MC3R gene in obese Polish children and adolescents. Mol Biol Rep. 2013;40(12):6893-6898.

25. Farooqi IS, et al. Dominant and recessive inheritance of morbid obesity associated with melanocortin 4 receptor deficiency. J Clin Invest. 2000;106(2):271-279.

26. Krude H, Biebermann H, Luck W, Horn R, Brabant $G$, Grüters A. Severe early-onset obesity, adrenal insufficiency and red hair pigmentation caused by POMC mutations in humans. Nat Genet. 1998;19(2):155-157.

27. Dubern B, Clement K. Leptin and leptin receptor-related monogenic obesity. Biochimie. 2012;94(10):2111-2115.

28. Clément K, et al. A mutation in the human leptin receptor gene causes obesity and pituitary dysfunction. Nature. 1998;392(6674):398-401.

29. Montague CT, et al. Congenital leptin deficiency is associated with severe early-onset obesity in humans. Nature. 1997;387(6636):903-908.

30. Mouse Genome Sequencing Consortium, et al. Initial sequencing and comparative analysis of the mouse genome. Nature. 2002;420(6915):520-562.

31. Kile BT, Hilton DJ. The art and design of genetic screens: mouse. Nat Rev Genet. 2005;6(7):557-567.

32. Ding S, Wu X, Li G, Han M, Zhuang Y, Xu T. Efficient transposition of the piggyBac (PB) transposon in mammalian cells and mice. Cell. 2005;122(3):473-483.

33. Sun LV, et al. PBmice: an integrated database system of piggyBac (PB) insertional mutations and their characterizations in mice. Nucleic Acids Res. 2008;36(Database issue):D729-D734.

34. Danilovich N, Babu PS, Xing W, Gerdes M, Krishnamurthy H, Sairam MR. Estrogen deficiency, obesity, and skeletal abnormalities in follicle-stimulating hormone receptor knockout (FORKO) female mice. Endocrinology 2000;141(11):4295-4308.

35. Kawasawa Y, Kume K, Nakade S, Haga H, Izumi T, Shimizu T. Brain-specific expression of novel G-protein-coupled receptors, with homologies to Xenopus PSP24 and human GPR45. Biochem Biophys Res Commun. 2000;276(3):952-956.

36. Maffei M, et al. Leptin levels in human and rodent: measurement of plasma leptin and ob RNA in obese and weight-reduced subjects. Nat Med.1995;1(11):1155-1161.

37. Considine RV, et al. Serum immunoreactiveleptin concentrations in normal-weight and obese humans. N Engl JMed. 1996;334(5):292-295.

38. Reaven GM. Banting lecture 1988. Role of insulin resistance in human disease. Diabetes. 1988;37(12):1595-1607.

39. Kahn SE, Hull RL, Utzschneider KM. Mechanisms linking obesity to insulin resistance and type 2 diabetes. Nature. 2006;444(7121):840-846

40. Kim JK, et al. Redistribution of substrates to adipose tissue promotes obesity in mice with selective insulin resistance in muscle. J Clin Invest. 2000;105(12):1791-1797.

41. Brüning JC, et al. A muscle-specific insulin receptor knockout exhibits features of the metabolic syndrome of NIDDM without altering glucose tolerance. Mol Cell. 1998;2(5):559-569.

42. Lonardo A, Carani C, Carulli N, Loria P. 'Endo- crine NAFLD' a hormonocentric perspective of nonalcoholic fatty liver disease pathogenesis. J Hepatol. 2006;44(6):1196-1207.

43. Fabbrini E, et al. Intrahepatic fat, not visceral fat, is linked with metabolic complications of obesity. Proc Natl Acad Sci U S A 2009;106(36):15430-15435.

44. Rosen ED, Spiegelman BM. Adipocytes as regulators of energy balance and glucose homeostasis. Nature. 2006;444(7121):847-853.

45. Garland T, et al. The biological control of voluntary exercise, spontaneous physical activity and daily energy expenditure in relation to obesity: human and rodent perspectives. J Exp Biol. 2011;214(Pt 2):206-229.

46. Pugh PL, Ahmed SF, Smith MI, Upton N, Hunter AJ. A behavioural characterisation of the FVB/N mouse strain. Behav Brain Res. 2004;155(2):283-289.

47. Cowley MA, et al. Leptin activates anorexigenic POMC neurons through a neural network in the arcuate nucleus. Nature. 2001;411(6836):480-484.

48. Bunel DT, Delbende C, Blasquez C, Jégou S, Vaudry H. Effects of ions and ionic channel activators or blockers on release of alpha-MSH from perifused rat hypothalamic slices. Brain Res Mol Brain Res. 1990;8(2):167-175.

49. de Souza FS, et al. Identification of neuronal enhancers of the proopiomelanocortin gene by transgenic mouse analysis and phylogenetic footprinting. Mol Cell Biol. 2005;25(8):3076-3086.

50. Münzberg H, Huo L, Nillni EA, Hollenberg AN, Bjørbaek C. Role of signal transducer and activator of transcription 3 in regulation of hypothalamic proopiomelanocortin gene expression by leptin. Endocrinology. 2003;144(5):2121-2131.

51. Xu AW, Ste-Marie L, Kaelin CB, Barsh GS. Inactivation of signal transducer and activator of transcription 3 in proopiomelanocortin (Pomc) neurons causes decreased pomc expression, mild obesity, and defects in compensatory refeeding. Endocrinology. 2007;148(1):72-80.

52. Gao Q, et al. Disruption of neural signal transducer and activator of transcription 3 causes obesity, diabetes, infertility, and thermal dysregulation. Proc Natl Acad Sci U S A 2004;101(13):4661-4666.

53. Silva VR, et al. Hypothalamic S1P/S1PR1 axis controls energy homeostasis. Nat Commun. 2014;5:4859.

54. Marrero MB, Venema VJ, Ju H, Eaton DC, Venema RC. Regulation of angiotensin II-induced JAK2 tyrosine phosphorylation: roles of SHP-1 and SHP2. Am J Physiol. 1998;275(5 Pt 1):C1216-C1223.

55 . Godeny MD, et al. The N-terminal SH2 domain of the tyrosine phosphatase, SHP-2, is essential for Jak2-dependent signaling via the angiotensin II type AT1 receptor. Cell Signal. 2007;19(3):600-609.

56. Lukashova V, Chen Z, Duhé RJ, Rola-Pleszczynski M, Stanková J. Janus kinase 2 activation by the platelet-activating factor receptor (PAFR): roles of Tyk2 and PAFR C terminus. J Immunol. 2003;171(7):3794-3800.

57. Henry FE, Sugino K, Tozer A, Branco T, Sternson SM. Cell type-specific transcriptomics of hypothalamic energy-sensing neuron responses to weight-loss. Elife. 2015;4:e09800.

58. Forss-Petter S, et al. Transgenic mice expressing beta-galactosidase in mature neurons under neuron-specific enolase promoter control. Neuron. 1990;5(2):187-197.

59. Tung YC, Piper SJ, Yeung D, O'Rahilly S, Coll AP. A comparative study of the central effects of specific proopiomelancortin (POMC)-derived melanocortin peptides on food intake and body weight in pomc null mice. Endocrinology. 2006;147(12):5940-5947.

60. Kawasawa Y, Kume K, Izumi T, Shimizu T. Mammalian PSP24s (alpha and beta isoforms) are not responsive to lysophosphatidic acid in mammalian expression systems. Biochem Biophys Res Commun. 2000;276(3):957-964.

61. Rosenbaum DM, Rasmussen SG, Kobilka BK. The structure and function of G-protein-coupled receptors. Nature. 2009;459(7245):356-363.

62. Rask-Andersen M, Almén MS, Schiöth HB Trends in the exploitation of novel drug targets. Nat Rev Drug Discov. 2011;10(8):579-590.

63. Bumaschny VF, et al. Obesity-programmed mice are rescued by early genetic intervention. JClin Invest. 2012;122(11):4203-4212.

64. McGuinness OP, Ayala JE, Laughlin MR, Wasserman DH. NIH experiment in centralized mouse phenotyping: the Vanderbilt experience and recommendations for evaluating glucose homeostasis in the mouse. Am J Physiol Endocrinol Metab. 2009;297(4):E849-E855.

65. Williams KW, et al. Segregation of acute leptin and insulin effects in distinct populations of arcuate proopiomelanocortin neurons. J Neurosci. 2010;30(7):2472-2479.

66. Cheung CC, Clifton DK, Steiner RA. Proopiomelanocortin neurons are direct targets for leptin in the hypothalamus. Endocrinology. 1997;138(10):4489-4492.

67. Sohn JW, Xu Y, Jones JE, Wickman K, Williams KW, Elmquist JK. Serotonin $2 \mathrm{C}$ receptor activates a distinct population of arcuate pro-opiomelanocortin neurons via TRPC channels. Neuron. 2011;71(3):488-497.

68. Soskis MJ, et al. A chemical genetic approach reveals distinct EphB signaling mechanisms during brain development. Nat Neurosci. 2012;15(12):1645-1654.

69. Tasaki T, et al. Biochemical and genetic studies of UBR3, a ubiquitin ligase with a function in olfactory and other sensory systems. J Biol Chem. 2007;282(25):18510-18520.

70. Günther T, et al. Genetic ablation of parathyroid glands reveals another source of parathyroid hormone. Nature. 2000;406(6792):199-203

71. Schreiber J, et al. Placental failure in mice lacking the mammalian homolog of glial cells missing, GCMa. Mol Cell Biol. 2000;20(7):2466-2474.

72. Stehling O, et al. Human CIA2A-FAM96A and CIA2B-FAM96B integrate iron homeostasis and maturation of different subsets of cytosolic-nuclear iron-sulfur proteins. Cell Metab. 2013;18(2):187-198.

73. Miller AL, Flecknell PA, Leach MC, Roughan JV. A comparison of a manual and an automated behavioural analysis method for assessing postoperative pain in mice. Appl Anim Behav Sci. 2011;131:138-144. 
74. Masuya H, et al. Implementation of the modifiedSHIRPA protocol for screening of dominant phenotypes in a large-scale ENU mutagenesis program. Mamm Genome. 2005;16(11):829-837.

75. Lei K, et al. SUN1 and SUN2 play critical but partially redundant roles in anchoring nuclei in skeletal muscle cells in mice. Proc Natl Acad Sci U S A. 2009;106(25):10207-10212.
76. Kitamura T, et al. Forkhead protein FoxO1 mediates Agrp-dependent effects of leptin on food intake. Nat Med. 2006;12(5):534-540.

77. Cepoi D, et al. Assessment of a small molecule melanocortin-4 receptor-specific agonist on energy homeostasis. Brain Res. 2004; $1000(1-2): 64-71$.

78. Xue L, et al. Most vesicles in a central nerve terminal participate in recycling. J Neurosci. 2013;33(20):8820-8826.

79. Sun ZC, et al. Extremely low frequency electromagnetic fields facilitate vesicle endocytosis by increasing presynaptic calcium channel expression at a central synapse. Sci Rep. 2016;6:21774.

80. Tschöp MH, et al. A guide to analysis of mouse energy metabolism. Nat Methods. 2012;9(1):57-63. 